\title{
Predicting brain atrophy from tau pathology: a summary of clinical findings and their translation into personalized models
}

\author{
Amelie Schäfer *,1, a, Pavanjit Chaggar ${ }^{1, b}$, Travis B. Thompson ${ }^{\mathrm{b}}$, Alain Goriely ${ }^{2, \mathrm{~b}}$, Ellen Kuhl ${ }^{\mathrm{a}}$, \\ for the Alzheimer's Disease Neuroimaging Initiative ${ }^{3}$ \\ ${ }^{\text {a }}$ Living Matter Laboratory, Stanford University, Stanford, California, USA \\ ${ }^{\mathrm{b}}$ Mathematical Institute, University of Oxford, Oxford, United Kingdom
}

\section{A B S T R A C T}

For more than 25 years, the amyloid hypothesis-the paradigm that amyloid is the primary cause of Alzheimer's disease-has dominated the Alzheimer's community. Now, increasing evidence suggests that tissue atrophy and cognitive decline in Alzheimer's disease are more closely linked to the amount and location of misfolded tau protein than to amyloid plaques. However, the precise correlation between tau pathology and tissue atrophy remains unknown. Here we integrate multiphysics modeling and Bayesian inference to create personalized tau-atrophy models using longitudinal clinical images from the Alzheimer's Disease Neuroimaging Initiative. For each subject, we infer three personalized parameters, the tau misfolding rate, the tau transport coefficient, and the tau-induced atrophy rate from four consecutive annual tau positron emission tomography scans and structural magnetic resonance images. Strikingly, the tau-induced atrophy coefficient of $0.13 /$ year (95\% CI: 0.097-0.189) was fairly consistent across all subjects suggesting a strong correlation between tau pathology and tissue atrophy. Our personalized whole brain atrophy rates of 0.68-1.68\%/year (95\% CI: 0.5-2.0) are elevated compared to healthy subjects and agree well with the atrophy rates of $1-3 \%$ /year reported for Alzheimer's patients in the literature. Once comprehensively calibrated with a larger set of longitudinal images, our model has the potential to serve as a diagnostic and predictive tool to estimate future atrophy progression from clinical tau images on a personalized basis.

Statement of Significance: Developing predictive, patient-specific models of Alzheimer's disease pathology and progression is of paramount importance for effective patient care and potential treatment. Tissue atrophy, the reduction of brain volume, is an important biomarker for Alzheimer's disease. Similarly, the pathology associated with tau proteins is thought to play a central role in Alzheimer's disease progression, local atrophy, and a patient's cognitive decline. The main question is: how do combine the mechanisms of tau propagation and atrophy in a single model that can make the best use of existing data? Here, we first review the dynamics of atrophy in for Alzheimer's disease and describe a mathematical model that couples tau propagation and atrophy. We then investigate how to fit the model parameters using the longitudinal structural neuroimaging data of four subjects, from the ADNI database, and a Bayesian Markov Chain Monte Carlo inference method. Our approach shows that network neurodegeneration models may hold promise for the predictive, patient-specific modeling of AD using AV-1451 tau PET and T1 structural MRI data.

\section{Introduction}

Alzheimer's disease is associated with the production and propagation of misfolded oligomers of amyloid- $\beta$ and tau proteins which have been found to accumulate in the form of amyloid- $\beta$ plaques and tau neurofibrillary tangles in the brains of patients [1]. Aggregated plaques of amyloid oligomers outside of neuronal cells can impair neuronal function at the synapses and cause neuroinflammation through the activation of microglial cells $[2,3]$. Hyperphosphorylated tau protein is produced inside neuronal cells and aggregates in neurofibrillary tangles that disrupt the axonal transport of nutrients [4]. Fig. 1 illustrates the molecular level neuropathology of Alzheimer's disease. The early amyloid cascade hypothesis posits that amyloid aggregates are the primary catalyst of Alzheimer's disease and its related cognitive decline [5-7]. In recent years however, the focus has shifted to hyperphosphorylated tau protein as a main agent in disease progression, as multiple studies have found a direct correlation between the amount and distribution of misfolded tau protein and cognitive impairment in Alzheimer's patients

\footnotetext{
* Corresponding author.

E-mail address: amesch@stanford.edu (A. Schäfer).

1 Joint first author

2 Senior Author

${ }^{3}$ Data used in preparation of this article were obtained from the Alzheimer's Disease Neuroimaging Initiative (ADNI) database (adni.loni.usc.edu). As such, the investigators within the ADNI contributed to the design and implementation of ADNI and/or provided data but did not participate in analysis or writing of this report. A complete listing of ADNI investigators can be found at: http://adni.loni.usc.edu/wp-content/uploads/how_to_apply/ADNI_Acknowledgement_List.pdf
} 
[8-12]. Similarly, there appears to be a strong association of pathological tau with tissue atrophy. Tau pathology and cortical atrophy have been observed to follow the same stereotypical spatiotemporal pattern [13-16], and tau positron emission tomography (PET) signal has been found to be a more reliable predictor for atrophy than either amyloid PET or baseline cortical thickness [11].

Mathematical modeling represents a promising, non-invasive means of investigating the mechanisms and characteristics of neurodegenerative diseases. Due to its direct correlation with tissue atrophy and cognitive symptoms in Alzheimer's disease, tau pathology is a specifically interesting target for modeling, as it bears high potential for the prediction of disease progression timelines and the evaluation of potential future treatments. Several studies have focused on models for tau pathology and their validation with cross-sectional or longitudinal patient data [17-19]. We have previously proposed a network diffusion model to simulate misfolded tau protein propagation and shown that it can capture individual patient pathology by learning its model parameters from tau PET data of 76 subjects using Bayesian inference [20]. Similarly, several mathematical models have been used to shed light on the potential interplay between atrophy dynamics and the prion-like progression of misfolded tau deposition [21-24]. Yet, to date, there is no physics-based model that integrates atrophy dynamics and Bayesian learning with multimodal neuroimaging data of both tau pathology and tissue atrophy.

Here we focus on two primary objectives: First, we conduct a brief review of atrophy dynamics in Alzheimer's disease which motivates our physics-based network diffusion model of coupled tau progression and atrophy evolution. Second, we perform an inaugural Bayesian inference study to personalize its tau and atrophy parameters. This study lays the foundation for larger, predictive network atrophy studies and for the further development of more complex coupled tau and atrophy models. The remainder of the manuscript is organized as follows: In Section 2, we review literature on how atrophy manifests in Alzheimer's disease and establish a link between the progression of atrophy and the spatiotemporal evolution of misfolded tau protein. Section 3 introduces a network proteopathy model augmented with an atrophy model motivated by the literature. In Section 4, we present experimental tau and atrophy data for four subjects from the Alzheimer's Disease Neuroimaging Initiative [25] and demonstrate how to personalize the model using a probabilistic Bayesian approach. We discuss the features of our model in Sections 5 and 6, and offer concluding remarks and practical perspectives in Section 7.

\section{Brain atrophy in Alzheimer's disease}

In this section, we summarize the characteristic clinical features of atrophy in Alzheimer's disease, its temporal evolution, and its correlation to hyperphosphorylated tau protein. These observations lay the foundation for our physics-based models that couple the spatiotemporal evolution of tau pathology and tissue atrophy in Section 3.

\subsection{Characteristics of atrophy}

Tissue atrophy is a classical hallmark of neurodegenerative diseases. In Alzheimer's disease, atrophy is regionally heterogeneous and presents in the form of volume loss, morphological changes, and cortical thinning in the gray matter of the brain $[14,16,26-28]$, typically accompanied by white matter lesions [29], white matter tract changes [30,31], and enlargement of the lateral ventricles [32]. The stereotypical spatiotemporal sequence of atrophy changes in the neocortex aligns with the topographic pattern of neurofibrillary tau tangles $[13,14]$. Usually, volume loss is first observed in the medial temporal lobe, including the hippocampus and the entorhinal cortex, two structures whose volume changes are established biomarkers for Alzheimer's disease progression [33]. With advancing disease, an increasing number of neocortical regions is affected by atrophy, initially the lateral temporal lobe, subsequently the parietal and frontal lobes. The sensorimotor and visual cortices are typically spared from atrophy until late in the disease [15]. Fig. 1 illustrates the organ level neuropathology of Alzheimer's disease: volume loss in the hippocampus, cortical thinning, morphological changes like the widening of cortical sulci, and the enlargement of the lateral ventricles as a consequence of a volume loss in adjacent gray matter structures.

\subsection{Dynamics of atrophy}

Numerous brain imaging studies have investigated the characteristics of atrophy evolution in the pre-clinical and clinical phases of Alzheimer's disease in comparison to healthy aging. These studies are commonly based on longitudinal magnetic resonance imaging (MRI) of a cohort of subjects combined with longitudinal cognitive assessments. Regional and global brain volume changes are evaluated from serial MRIs using a multitude of automated and semi-automated algorithms for image registration, segmentation, and morphometry.

Atrophy rates are higher in pre-symptomatic and symptomatic Alzheimer's patients than in healthy controls. Throughout the

\section{healthy brain Alzheimer's disease brain}

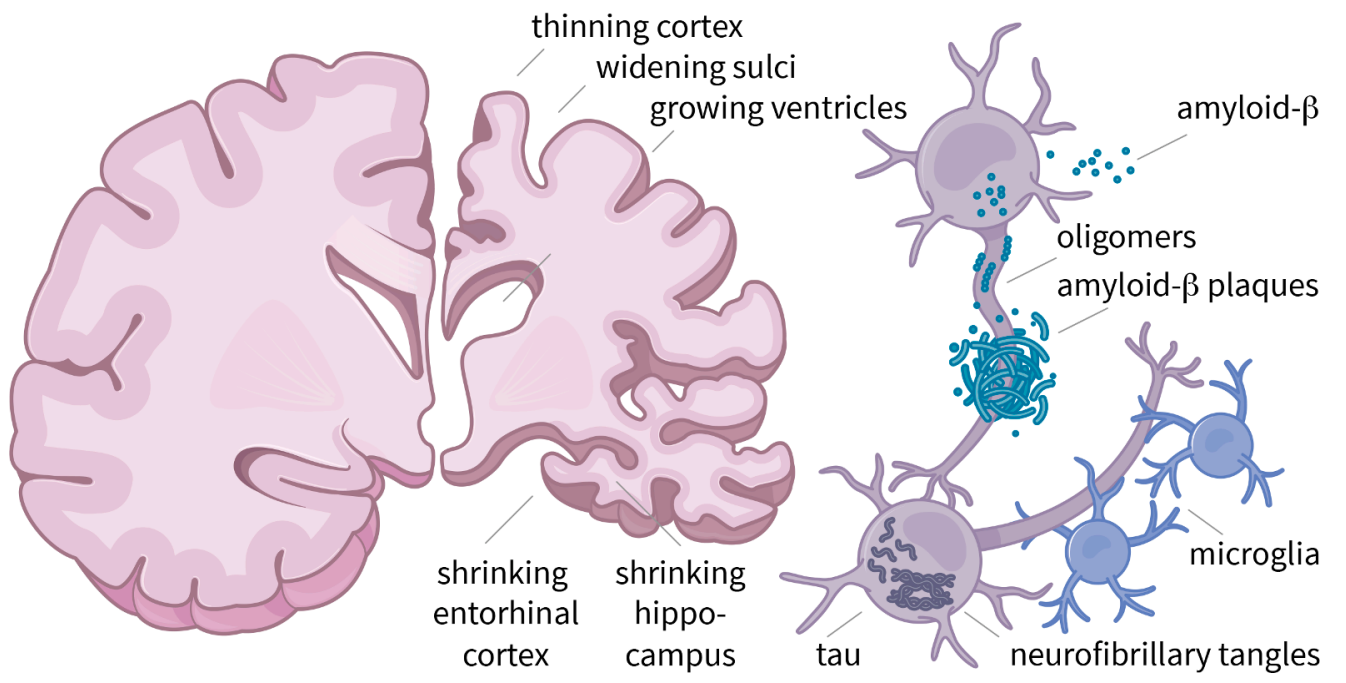

Fig. 1. Neuropathology of Alzheimer's disease. Amyloid- $\beta$ peptides are produced by the cleavage of amyloid precursor protein in the membrane of neurons. In the extracellular space, amyloid- $\beta$ peptides form flexible soluble oligomers. Fibers of amyloid- $\beta$ oligomers assemble into amyloid- $\beta$ plaques, which interfere with neural function. Plaque formation triggers microglial activation and neuroinflammation. Inside the neurons, hyperphosphorylated tau proteins form neurofibrillary tangles, which displace organelles and disrupt vesicular transport. Neurons die and cause brain atrophy. Atrophy manifests itself in a thinning of the cerebral cortex, a widening of the cortical sulci, a growing of the ventricles, a shrinkage of the entorhinal cortex and the hippocampus, and a marked loss in brain volume. Ultimately, brain atrophy results in a progressive cognitive decline. 
course of a lifespan, all human brains exhibit a certain amount of tissue atrophy [34-36]. Increased cerebral atrophy is a widely-accepted biomarker for Alzheimer's disease. Patients diagnosed with Alzheimer's disease or mild cognitive impairment (MCI) show elevated rates of cortical thinning and volume loss in the hippocampus and across the whole brain compared to cognitively normal older adults [37,38]. A study that compared hippocampal atrophy rates in mutation carriers for familial Alzheimer's disease and non-carriers found that atrophy rates were significantly higher in mutation carriers, even before the onset of cognitive symptoms, and that the difference between the two groups

Table 1

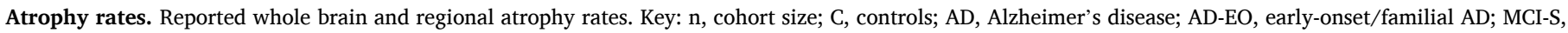

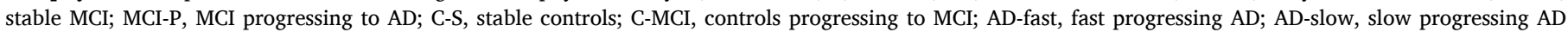
[51-55].

\begin{tabular}{|c|c|c|c|c|}
\hline study & \multicolumn{2}{|c|}{ subjects } & $\begin{array}{l}\text { atrophy rate in mean (SD) } \\
\% / \text { year unless specified }\end{array}$ & other observations \\
\hline & & & \multicolumn{2}{|l|}{ whole brain } \\
\hline Chan et al. (2003) [51] & $\mathrm{AD}-\mathrm{EO}$ & $\mathrm{n}=12$ & $2.8(95 \%$ CI $2.3-3.3)$ & annual volume loss rises by $0.32 \% /$ year $^{2}$ \\
\hline Ridha et al. (2006) [39] & $\begin{array}{l}\text { C-S } \\
\text { C-MCI } \\
\text { MCI-P } \\
\text { AD-EO }\end{array}$ & $\begin{array}{l}\mathrm{n}=25 \\
\mathrm{n}=5 \\
\mathrm{n}=6 \\
\mathrm{n}=5\end{array}$ & $\begin{array}{l}0.0(0.57) \\
0.52(0.51) \\
1.82(0.87) \\
1.79(0.74)\end{array}$ & $\begin{array}{l}\text { atrophy rates significantly higher in mutation } \\
\text { carriers compared to controls, discrepancy in- } \\
\text { creases over time }\end{array}$ \\
\hline Sluimer et al. (2008) [48] & $\begin{array}{l}\mathrm{AD}-\mathrm{EO} \\
\mathrm{AD}\end{array}$ & $\begin{array}{l}\mathrm{n}=26 \\
\mathrm{n}=39\end{array}$ & $\begin{array}{l}2.2(1.1) \\
1.7(0.8)\end{array}$ & higher atrophy rates in early-onset $\mathrm{AD}$ \\
\hline Jack et al. (2004) [52] & $\begin{array}{l}\mathrm{C} \\
\mathrm{C}-\mathrm{MCI} \\
\text { AD-slow } \\
\text { AD-fast }\end{array}$ & $\begin{array}{l}\mathrm{n}=40 \\
\mathrm{n}=15 \\
\mathrm{n}=32 \\
\mathrm{n}=33\end{array}$ & $\begin{array}{l}0.4(0.3) \\
0.8(0.5) \\
0.6(0.7) \\
1.4(1.1)\end{array}$ & $\begin{array}{l}\text { atrophy rates are higher in subjects who progress } \\
\text { from } \mathrm{C} \text { to } \mathrm{MCI} \text { than in non-progressors, and } \\
\text { higher in } \mathrm{AD} \text { with fast cognitive decline than in } \\
\text { subjects with slow cognitive decline }\end{array}$ \\
\hline Henneman et al. (2009) [42] & $\begin{array}{l}\mathrm{C} \\
\mathrm{MCI} \\
\mathrm{AD}\end{array}$ & $\begin{array}{l}\mathrm{n}=34 \\
\mathrm{n}=44 \\
\mathrm{n}=64\end{array}$ & $\begin{array}{l}0.6(0.6) \\
1.3(0.9) \\
1.9(0.9)\end{array}$ & $\begin{array}{l}\text { significant difference in atrophy rates between } \\
\text { groups (C, MCI, AD) }\end{array}$ \\
\hline \multirow[t]{2}{*}{ Leung et al. (2013) [47] } & $\begin{array}{l}\mathrm{C} \\
\mathrm{MCI} \\
\mathrm{AD}\end{array}$ & $\begin{array}{l}\mathrm{n}=205 \\
\mathrm{n}=352 \\
\mathrm{n}=156\end{array}$ & $\begin{array}{l}0.63(95 \% \text { CI } 0.53-0.72) \\
1.01(95 \% \text { CI } 0.92-1.1) \\
1.41(95 \% \text { CI } 1.23-1.59)\end{array}$ & $\begin{array}{l}\text { acceleration in atrophy rates between groups (C, } \\
\text { MCI, AD) but not evidence of acceleration within } \\
\text { groups }\end{array}$ \\
\hline & & & \multicolumn{2}{|l|}{ hippocampus } \\
\hline Barnes et al. (2008) [53] & $\begin{array}{l}\mathrm{C} \\
\mathrm{AD}\end{array}$ & $\begin{array}{l}\mathrm{n}=212 \\
\mathrm{n}=595\end{array}$ & $\begin{array}{l}1.41(95 \% \text { CI } 0.52-2.3) \\
4.66(95 \% \text { CI } 3.92-5.4)\end{array}$ & $\begin{array}{l}\text { meta-analysis of nine studies on hippocampal at- } \\
\text { rophy rates }\end{array}$ \\
\hline Henneman et al. (2009) [42] & $\begin{array}{l}\mathrm{C} \\
\mathrm{MCI} \\
\mathrm{AD}\end{array}$ & $\begin{array}{l}\mathrm{n}=34 \\
\mathrm{n}=44 \\
\mathrm{n}=64\end{array}$ & $\begin{array}{l}2.2(1.4) \\
3.8(1.2) \\
4.0(1.2)\end{array}$ & $\begin{array}{l}\text { significant difference in atrophy rates between } \\
\text { groups (C, MCI, AD) }\end{array}$ \\
\hline Leung et al. (2013) [47] & $\begin{array}{l}\mathrm{C} \\
\mathrm{MCI} \\
\mathrm{AD}\end{array}$ & $\begin{array}{l}\mathrm{n}=205 \\
\mathrm{n}=352 \\
\mathrm{n}=156\end{array}$ & $\begin{array}{l}1.22(95 \% \text { CI } 0.9-1.55) \\
3.20(95 \% \text { CI } 2.82-3.57) \\
5.19(95 \% \text { CI } 4.44-5.94)\end{array}$ & $\begin{array}{l}\text { hippocampal atrophy rates accelerate by } 0.22 \\
\% / \text { year }^{2}\end{array}$ \\
\hline Jack et al. (2004) [52] & $\begin{array}{l}\text { C } \\
\text { C-MCI } \\
\text { AD-slow } \\
\text { AD-fast }\end{array}$ & $\begin{array}{l}\mathrm{n}=40 \\
\mathrm{n}=15 \\
\mathrm{n}=32 \\
\mathrm{n}=33\end{array}$ & $\begin{array}{l}1.4(1.2) \\
3.3(2.4) \\
3.0(4.5) \\
3.6(3.2)\end{array}$ & $\begin{array}{l}\text { atrophy rates are higher in subjects who progress } \\
\text { from } C \text { to } M C I \text { than in non-progressors, and } \\
\text { higher in } A D \text { with fast cognitive decline than in } \\
\text { subjects with slow cognitive decline }\end{array}$ \\
\hline \multirow[t]{2}{*}{ McDonald et al. (2009) [45] } & $\begin{array}{l}\mathrm{C} \\
\mathrm{MCI} \\
\mathrm{AD}\end{array}$ & $\begin{array}{l}\mathrm{n}=137 \\
\mathrm{n}=231 \\
\mathrm{n}=104\end{array}$ & $\begin{array}{l}0.86(1.27) \\
2.39(2.26) \\
3.64(2.12)\end{array}$ & $\begin{array}{l}\text { atrophy rates in all neocortical regions accelerate } \\
\text { with clinical impairment in } \mathrm{AD} \text {, atrophy is not } \\
\text { linear or uniform across regions }\end{array}$ \\
\hline & & & \multicolumn{2}{|l|}{ entorhinal cortex } \\
\hline Jack et al. (2004) [52] & $\begin{array}{l}\mathrm{C} \\
\mathrm{C}-\mathrm{MCI} \\
\text { AD-slow } \\
\text { AD-fast }\end{array}$ & $\begin{array}{l}\mathrm{n}=40 \\
\mathrm{n}=15 \\
\mathrm{n}=32 \\
\mathrm{n}=33\end{array}$ & $\begin{array}{l}2.9(2.6) \\
5.1(5.1) \\
8.0(5.8) \\
8.4(9.2)\end{array}$ & \\
\hline Miller et al. (2013) [54] & $\begin{array}{l}\mathrm{C} \\
\mathrm{C}-\mathrm{MCI}\end{array}$ & $\begin{array}{l}\mathrm{n}=81 \\
\mathrm{n}=20\end{array}$ & $\begin{array}{l}1.0(3.3) \\
2.7(3.1)\end{array}$ & \\
\hline \multirow[t]{2}{*}{ McDonald et al. (2009) [45] } & $\begin{array}{l}\mathrm{C} \\
\mathrm{MCI} \\
\mathrm{AD}\end{array}$ & $\begin{array}{l}\mathrm{n}=137 \\
\mathrm{n}=231 \\
\mathrm{n}=104\end{array}$ & $\begin{array}{l}0.52(1.13) \\
1.93(1.56) \\
2.50(1.13)\end{array}$ & $\begin{array}{l}\text { atrophy rates in all neocortical regions accelerate } \\
\text { with clinical impairment in } \mathrm{AD} \text {, atrophy is not } \\
\text { linear or uniform across regions }\end{array}$ \\
\hline & & & \multicolumn{2}{|l|}{ medial temporal lobe } \\
\hline Sluimer et al. (2009) [44] & $\begin{array}{l}\mathrm{C} \\
\mathrm{MCI} \\
\mathrm{AD}\end{array}$ & $\begin{array}{l}\mathrm{n}=34 \\
\mathrm{n}=44 \\
\mathrm{n}=64\end{array}$ & $\begin{array}{l}0.6(0.7) \\
1.5(0.7) \\
1.5(0.7)\end{array}$ & $\begin{array}{l}\text { atrophy rate in medial temporal lobe already } \\
\text { reaches maximum before transition from MCI to } \\
\mathrm{AD}\end{array}$ \\
\hline \multirow[t]{2}{*}{ Rusinek et al. (2004) [55] } & $\begin{array}{l}\mathrm{C} \\
\mathrm{AD}\end{array}$ & $\begin{array}{l}\mathrm{n}=21 \\
\mathrm{n}=18\end{array}$ & $\begin{array}{l}0.37(0.34) \\
\text { left: } 2.2 \text {, accel. } 0.73 \% / \text { year }^{2} \\
\text { right: } 1.8 \text {, accel. } 0.34 \% / \text { year }^{2}\end{array}$ & $\begin{array}{l}\text { annual atrophy rate increases with disease pro- } \\
\text { gression in AD }\end{array}$ \\
\hline & & & ventricular expansion & \\
\hline Jack et al. (2004) [52] & $\begin{array}{l}\text { C } \\
\text { C-MCI } \\
\text { AD-slow } \\
\text { AD-fast }\end{array}$ & $\begin{array}{l}\mathrm{n}=40 \\
\mathrm{n}=15 \\
\mathrm{n}=32 \\
\mathrm{n}=33\end{array}$ & $\begin{array}{l}1.7(0.9) \\
3.4(1.6) \\
4.3(3.3) \\
6.4(3.7)\end{array}$ & $\begin{array}{l}\text { expansion rates are higher in subjects who } \\
\text { progress from } C \text { to } M C I \text { than in non-progressors, } \\
\text { and higher in AD with fast cognitive decline than } \\
\text { in subjects with slow cognitive decline }\end{array}$ \\
\hline Leung et al. (2013) [47] & $\begin{array}{l}\mathrm{C} \\
\mathrm{MCI} \\
\mathrm{AD}\end{array}$ & $\begin{array}{l}\mathrm{n}=205 \\
\mathrm{n}=352 \\
\mathrm{n}=156\end{array}$ & $\begin{array}{l}1.34 \mathrm{~mL} / \text { year }(95 \% \text { CI } 1.09-1.6) \\
2.69 \mathrm{~mL} / \text { year }(95 \% \text { CI } 2.38-3.01) \\
4.04 \mathrm{~mL} / \text { year }(95 \% \text { CI } 3.34-4.75)\end{array}$ & $\begin{array}{l}\text { rate of ventricular expansion accelerates in MCI } \\
\text { and } A D \text { subjects }\end{array}$ \\
\hline
\end{tabular}


increased over time [39]. Similarly, in a cohort of 240 subjects, whole brain atrophy rates were elevated for pre-symptomatic subjects who were later diagnosed with Alzheimer's disease within six years after conclusion of the image acquisition [40].

Atrophy rates accelerate with disease progression. A commonly observed characteristic of Alzheimer related atrophy is an accelerated volume loss over the course of the disease. For example, whole brain atrophy rates and rates of ventricular enlargement were found to increase as patients progress from MCI to Alzheimer's disease [41,42]. Whole brain atrophy rates were found to accelerate with age even in cognitively normal controls, but acceleration increased by a factor of 1.5 in Alzheimer's patients [43]. Two potentially intertwined explanations have been proposed for these accelerated whole brain atrophy rates: global acceleration could either stem from true acceleration of regional atrophy rates in already affected brain areas, or from the successive involvement of more brain regions as the disease progresses [41]. There is evidence of regional atrophy rates accelerating locally with disease severity when volume change is evaluated in different lobes [44] or even individual neocortical regions [45]. While atrophy rates appear to increase in all cortical regions with progressing cognitive impairment, the extent of local acceleration differs across areas [45].

Atrophy rates may decelerate at late disease stages. Observations of accelerating atrophy rates in Alzheimer's disease are ubiquitous, but some evidence points towards decelerating atrophy rates beyond a characteristic inflection point. In a study that compared global cortical thinning rates in cognitively normal, MCI, and Alzheimer's subjects, results indicate that atrophy rates first accelerate, but then decelerate with decreasing Mini Mental State Exam score. Even though data points from the low end of exam scores are sparse, the authors conclude that tissue atrophy follows a sigmoid like curve over the course of Alzheimer's disease with an inflection point around a Mini Mental State Exam score of 21 [37]. Another study identified decelerating hippocampal atrophy rates around five years before death while atrophy rates in middle frontal and inferior temporal lobes continued to accelerate until death [46]. A similar study found that atrophy rates in the middle temporal lobe, while increasing significantly from cognitively normal to mild cognitive impairment, did not increase further with transition to Alzheimer's disease. The study concludes that the middle temporal lobe might reach maximum atrophy rates already during mild cognitive impairment [44].

Alzheimer's related atrophy rates are higher in patients with early disease onset. In paradox to the general trend that atrophy rates tend to increase with age, imaging studies have consistently identified higher atrophy rates for younger symptomatic patients than for older patients $[41,47,48]$. This is consistent with the theory that Alzheimer's disease progresses more aggressively in patients with early disease onset $[49,50]$. Table 1 summarizes the observed regional and whole brain atrophy rates from multiple clinical studies. Most of the included studies offer a comparison between Alzheimer's patients at various stages of disease and cognitively normal, age-matched controls, providing evidence for increased atrophy rates in Alzheimer's disease and regional acceleration of atrophy rates with disease progression.

\subsection{Evidence relating atrophy to tau pathology}

Increasing evidence suggests that there is a close correlation between tau pathology and atrophy dynamics. In a study that combined longitudinal structural MRI within four years of death with postmortem neurofibrillary tangle autopsy, subjects with high tau burden showed higher gray matter loss in the medial and lateral temporal lobes compared to those with low tau burden [56]. Cross-sectional imaging studies have also shown that the pattern of neurodegeneration inferred from structural MRI mirrors the canonical progression of tau deposition in the brain $[9,13,14]$. Longitudinal imaging studies have consistently identified close correlations between regional tau PET signal intensity and a subsequent decrease in regional volume or cortical thickness in amyloid positive subjects and MCI or Alzheimer patients [10,12,57,58]. A recent study based on baseline amyloid and tau PET scans as well as baseline and follow-up structural MRIs for 32 patients during the early clinical stages of Alzheimer's disease, revealed the global tau PET intensity as a good predictor for future cortical thinning. Additionally, the results indicate a strong voxel-wise correlation between tau PET signal and future atrophy. Both on the global and local levels, tau PET was a stronger predictor for atrophy than amyloid PET or baseline cortical thickness [11].

\section{A network model for tau pathology and atrophy}

In this manuscript, we adapt the Fisher-Kolmogorov model [59] to characterize the local production and global spreading of misfolded tau protein across the brain. The continuous model is defined by

$\frac{\mathrm{d} c}{\mathrm{~d} t}=\operatorname{div}(\mathbf{K} \cdot \nabla c)+\alpha c[1-c]$

where $c$ denotes the concentration of misfolded tau protein, and $\mathbf{K}$ denotes the diffusion tensor that contains information about the speed and directionality of protein propagation. The growth coefficient $\alpha$ captures how much misfolded protein is produced or cleared locally. We discretize the Fisher-Kolmogorov model on a network that represents the structural brain connectome. Suppose that $G=\{E, N\}$ is the undirected graph of a structural brain connectome with edges $E$ and nodes $N$ that represent the anatomical regions of interest. A weighted adjacency matrix $\mathbf{W}$ is defined by the rule $W_{i j}=W_{j i}>0$ whenever $n_{i}$ is connected to $n_{j}$ by an edge $e_{i j}$ in $E$ and $W_{i j}=0$ otherwise. In this manuscript, we use an adjacency matrix constructed from diffusion tensor images of 426 participants of the human connectome project [60]. The network contains $N=83$ nodes, each representing a cortical or subcortical brain region. Each edge $e_{i j}$ of the graph represents a tractographic approximation of the white matter connections between two regions of interest and is associated with a measure of the average fiber number $n_{i j}$ and fiber length $\ell_{i j}$ along this connection. In line with a previous study [61], we define the weights of the adjacency matrix as $W_{i j}=n_{i j} / \ell_{i j}^{2}$. The files used to create the adjacency matrix are freely available $[62,63]$ as is the final adjacency matrix [64].

The weighted graph Laplacian $\mathbf{L}$ associated with the adjacency matrix $\mathbf{W}$ is then defined as

$\mathbf{L}=\mathbf{D}-\mathbf{W}$,

where $\mathbf{D}$ is a diagonal matrix defined by the row-sum of the weighted adjacency matrix as

$D_{i i}=\sum_{j=1}^{N} W_{i j} \quad$ with $\quad W_{i j}=n_{i j} / \ell_{i j}^{2}$.

The Laplacian allows us to discretize the model on the network,

$\frac{\mathrm{d} c_{i}}{\mathrm{~d} t}=-\rho \sum_{j=1}^{N} L_{i j} c_{j}+\alpha c_{i}\left[1-c_{i}\right]$,

where the quantity $c_{i}$ denotes the normalized concentration of toxic tau protein in regions $i=1,2, \cdots, N, \rho$ is a transport coefficient, $\alpha$ is a growth coefficient, and $L_{i j}$ are the entries of the weighted graph Laplacian.

Motivated by the observations of Section 2, we couple the tau evolution model of Equation (4) to an atrophy model of the following form,

$\frac{\mathrm{d} q_{i}}{\mathrm{~d} t}=\left[1-q_{i}\right] G_{c} c_{i}$,

and express atrophy in terms of a regional relative atrophy marker $0 \leq$ $q_{i} \leq 1$ for each node $i=1,2, \cdots, N$ in the connectome graph. The model postulates that the atrophy rate in a region of interest progresses based 
on the current atrophy level, $q_{i}$, and the current amount of misfolded tau, $c_{i}$, mitigated by a global tau-induced atrophy coefficient $G_{c}$. The dynamics of Equation (5) capture several known features of the atrophy dynamics discussed in Section 2. The direct dependency of the atrophy rate on the nodal protein concentration $c_{i}$, which itself follows a sigmoid curve over the course of the disease, allows us to capture the acceleration of atrophy rates observed in numerous longitudinal imaging studies. The saturation term $\left[1-q_{i}\right]$ in Equation (5) ensures an asymptotic deceleration of atrophy rates towards the later stages of the disease.

\section{Model personalization using medical images}

Our coupled tau-atrophy model contains three global parameters: a global transport coefficient $\rho$, a growth coefficient $\alpha$, and a tau-induced atrophy coefficient $G_{c}$. We have previously personalized the transport and growth coefficients $\rho$ and $\alpha$ of Equation (4) using Bayesian inference and longitudinal tau PET data [20]. Here we also include structural information from magnetic resonance images to personalize the atrophy coefficients $G_{c}$.

\subsection{Subject data}

We use longitudinal tau PET and structural imaging data from four subjects of the Alzheimer's Disease Neuroimaging Initiative database [25] to prototype our model personalization using Bayesian inference. Two subjects were classified as cognitively normal (CN), two were classified as late mild cognitively impaired (LMCI). All four subjects were previously identified as amyloid positive [65]. All four subjects received four consecutive annual AV-1451 tau PET and T1 structural MRI scans, with an average time of 1.08 years between scans.

Tau data preparation. The processing of the tau AV1451-PET data by ADNI followed standard protocols $[25,66]$. Briefly, for each subject, the PET images were co-registered to a corresponding high-resolution T1 weighted MRI, which had been segmented into 68 cortical and 15 subcortical regions according to the Desikan-Killiany atlas [67]. Each segmented region of interest corresponds to one node of our brain network from Section 3. For each region of interest, a PET standardized uptake value ratio was calculated using the inferior cerebellum as reference region. Since tau PET recordings in subcortical regions are known to be contaminated by off-target binding in the choroid plexus and nearby vascular structures [68-70], we exclude these regions from our analysis. To compare the clinically recorded PET tau values to our computationally simulated tau values, we map them into the range from zero to one. Briefly, we previously computed a regional tau positivity threshold of $c^{\text {raw }}=1.1$ based on fitting a two-component Gaussian mixture model to the raw tau PET data from a larger cohort of 76 subjects [20]. We set values below this threshold to zero and normalize the remaining values with respect to the maximum occurring standardized uptake value ratio in our previous study, such that the normalized values $c^{\text {pet }}$ range from zero to one, $0 \leq c^{\text {pet }} \leq 1$. For each subject, we set the initial conditions for the protein field in our model to the tau uptake values measured in the baseline PET scan $c^{\operatorname{sim}}(t=0)=c^{\text {pet }}\left(t_{0}\right)$.

Atrophy data preparation. We process the structural data using FreeSurfer [71] via the Clinica [72] t1-freesurfer-longitudinal pipeline. This image processing pipeline consists of a sequence of different tools of the FreeSurfer software and involves the computation of a subject-dependent template space, segmentation of subcortical structures, extraction of cortical surfaces, parcellation of cortical regions, cortical thickness estimation, and the extraction of volume and thickness estimates in the template space at different points in time. We compute the volumes for all 83 cortical and subcortical brain regions that coincide with the 83 nodes of our brain network model from Section 3 . We use the regional volumes measured at the baseline scan, $v_{0}^{\text {raw }}$, as reference points to which we normalize the regional volumes of all follow-up visits within each subject, $v^{\text {mri }}=v^{\text {raw }} / v_{0}^{\text {raw }}$. We then define the measured nodal atrophy as the relative reduction in volume, $q^{\mathrm{mri}}=1-v^{\mathrm{mri}}$, with an initial atrophy value of $q_{0}^{\mathrm{mri}}=1-v_{0}^{\mathrm{mri}}=0$. For each subject, we set the initial conditions for the atrophy field in our model to zero in all regions $q^{\operatorname{sim}}(t=0)=0$.

\subsection{Bayesian inference}

For each subject, we infer a personalized parameter set that most accurately explains the image data given the model in Equations (4) and (5). The parameter set $\boldsymbol{\vartheta}=\left\{\rho^{s}, \alpha^{s}, G_{c}^{s}\right\}$ consists of a personalized transport coefficient $\rho^{\mathrm{s}}$, growth coefficient $\alpha^{\mathrm{s}}$, and tau-induced atrophy coefficient $G_{c}^{\mathrm{s}}$. We approximate the posterior distribution $p(\vartheta \mid \widehat{D})$ of the parameters $\vartheta$ given the imaging data $\widehat{D}(t)$ using Bayesian inference,

$p(\boldsymbol{\vartheta} \mid \widehat{D}(t))=\frac{p(\widehat{D}(t) \mid \boldsymbol{\vartheta}) p(\boldsymbol{\vartheta})}{p(\widehat{D}(t))}$,

where $p(\widehat{D}(t) \mid \vartheta)$ is the likelihood, $p(\vartheta)$ are the priors, and $p(\widehat{D}(t))$ is the evidence.

For each subject, we specify a likelihood function that relates the computationally predicted tau and atrophy values at each region and time point, $\left\{c^{\text {sim }}\left(t_{i}\right), q^{\text {sim }}\left(t_{i}\right)\right\}$ to the clinically recorded tau and atrophy values, $\left\{c^{\text {pet }}\left(t_{i}\right), q^{\mathrm{mri}}\left(t_{i}\right)\right\}$. The time points $t_{i}$ for $i=0,1,2,3$ are associated with the four consecutive years at which the PET and structural scans were recorded for each subject. To account for clinical measurement noise, we assume that the likelihood between the clinically recorded data $\widehat{D}_{\mathrm{t}}(t)$ and $\widehat{D}_{\mathrm{a}}(t)$ and the computationally predicted data $D_{\mathrm{t}}(t, \vartheta)$ and $D_{\mathrm{a}}(t, \vartheta)$,

$p\left(\widehat{D}_{\mathrm{t}}(t) \mid \boldsymbol{\vartheta}\right) \sim \operatorname{Normal}\left(\right.$ mean $=D_{\mathrm{t}}(t, \boldsymbol{\vartheta})$, width $\left.=\sigma_{\mathrm{t}}\right)$

$p\left(\widehat{D}_{\mathrm{a}}(t) \mid \boldsymbol{\vartheta}\right) \sim \operatorname{Normal}\left(\right.$ mean $=D_{\mathrm{a}}(t, \boldsymbol{\vartheta})$, width $\left.=\sigma_{\mathrm{a}}\right)$.

is normally distributed around the computationally predicted data with likelihood widths of $\sigma_{\mathrm{t}}$ for tau and $\sigma_{\mathrm{a}}$ for atrophy.

For the priors, we select weakly informative prior distributions for all model parameters in the set $\vartheta=\left\{\rho^{\mathrm{s}}, \alpha^{\mathrm{s}}, G_{c}^{\mathrm{s}}\right\}$ in addition to priors for the noise parameters that dictate the likelihood widths $\sigma_{\mathrm{t}}^{\mathrm{s}}$ and $\sigma_{\mathrm{t}}^{\mathrm{a}}$. Table 2 summarizes the selection of our prior distributions.

With the likelihood from Equation (7) and the prior distributions from Table 2, we compute the posterior distributions $p\left(\vartheta \mid \widehat{D}_{\mathrm{t}}(t)\right)$ and $p\left(\vartheta \mid \widehat{D}_{\mathrm{a}}(t)\right)$ for the model parameters $\vartheta$ using Bayes' theorem,

$p\left(\boldsymbol{\vartheta} \mid \widehat{D}_{\mathrm{t}}(t)\right)=\frac{p\left(\widehat{D}_{\mathrm{t}}(t) \mid \boldsymbol{\vartheta}\right) p(\boldsymbol{\vartheta})}{p\left(\widehat{D}_{\mathrm{t}}(t)\right)} \quad$ and $p\left(\boldsymbol{\vartheta} \mid \widehat{D}_{\mathrm{a}}(t)\right)=\frac{p\left(\widehat{D}_{\mathrm{a}}(t) \mid \boldsymbol{\vartheta}\right) p(\boldsymbol{\vartheta})}{p\left(\widehat{D}_{\mathrm{a}}(t)\right)}$.

We adopt approximate inference techniques to numerically evaluate Equations (8) and personalize our model using the imaging data. We implement our model using two widely used Julia packages, the

Table 2

Prior distributions. Prior distributions for the personalized transport coefficient, growth coefficient, tau-induced atrophy coefficient, and the likelihood width.

\begin{tabular}{ll}
\hline Parameter & Distribution \\
\hline$\rho^{\mathrm{s}}$ & TruncatedNormal $\left(0<\rho^{\mathrm{s}}<10\right.$, mean $=0$, std $\left.=5\right)$ \\
$\alpha^{\mathrm{s}}$ & Normal $($ mean $=0, \operatorname{std}=5)$ \\
$G_{c}^{\mathrm{s}}$ & TruncatedNormal $\left(0<G_{c}^{\mathrm{s}}<10\right.$, mean $=0$, std $\left.=5\right)$ \\
$\sigma_{\mathrm{t}}^{\mathrm{s}}, \sigma_{\mathrm{a}}^{\mathrm{s}}$ & InverseGamma $($ shape $=2$, scale $=3)$ \\
\hline
\end{tabular}


DifferentialEquations library and the Turing probabilistic programming library. The DifferentialEquations library [73] allows us to solve the differential equations (4) and (5) in time, to produce the model predictions $D_{\mathrm{t}}(t, \boldsymbol{\vartheta})$ and $D_{\mathrm{a}}(t, \boldsymbol{\vartheta})$. The Turing probabilistic programming library [74] serves to implement a Bayesian Markov Chain Monte Carlo sampling approach. It supports several different samplers from which we select the No-U-Turn sampler [75] to design a Hamiltonian Markov Chain Monte Carlo method. For each subject, we sample five chains with 1000 tuning samples and 5000 posterior samples per chain.

For each subject, we calculate the posterior model predictions and credible intervals for the global tau and atrophy evolution by propagating the uncertainty of each model parameter's posterior distribution through our model. These predictions indicate how tau pathology and tissue atrophy may evolve over the next 30 years in each subject, based on the assumptions of our model and the information from the longitudinal imaging data.

\section{Results}

\subsection{Imaging data}

Fig. 2 provides an overview of the regional volume data for our set of four subjects. For subjects 1,3 , and 4 we observe on average monotonically decreasing volumes over time. However, a number of regions seem to either exhibit an increase or no change in volume compared to the baseline scan. In all four subjects, we observe a positive correlation between regional tau load and the average atrophy in a region which agrees well with the assumptions based on which we constructed our coupled tau-atrophy model. This correlation is stronger in subjects 1,2 , and 3 with Pearson correlation coefficients of $\mathrm{R}=0.382, \mathrm{R}=0.397$, and $\mathrm{R}=0.494$ respectively, than in subject 4 with $\mathrm{R}=0.144$. The regional volumes in subject 2 evolve less monotonically than those in the other subjects, and its average volume across all brain regions remains relatively constant over time. However, the plot shows that certain regions do decrease in volume compared to the baseline scan. Fig. 3 and Fig. 4 illustrate the collected tau and atrophy data on a template brain surface to show the regional distribution of the two fields in each subject.

\subsection{Posterior distributions}

Fig. 5 summarizes the personalized posterior distributions for the parameters of our coupled tau-atrophy model. All distributions have clearly moved from the weakly informative prior distributions, indicating successful inference. The estimates for measurement noise in the atrophy data $\sigma_{\mathrm{a}}$ are slightly higher than the estimated noise in the tau data $\sigma_{\mathrm{t}}$, especially for subjects 1 and 2 . The inferred transport coefficients $\rho$ for subjects 1,2 , and 4 are close to zero. Subject 3 seems to exhibit more pronounced tau dynamics, with an elevated transport coefficient $\rho$ and growth coefficient $\alpha$ compared to the rest of the cohort. Subject 4 represents an outlier compared to the other subjects when examining the inferred growth rate $\alpha$. The tau data from Subject 4 indicates a negative growth coefficient, which implies that there is more clearance than production of misfolded tau. The inferred tau-induced atrophy coefficients $G_{c}$ are of similar magnitude for all subjects.

\subsection{Posterior predictive simulations}

Fig. 6 shows the long- and short-term evolutions of global tau and atrophy predicted by our model for the four examined subjects. The tau and atrophy dynamics are best captured by our model for subject 3 . We note characteristic sigmoidal curves for normalized global tau and atrophy in the long-term predictions. All four data points for tau and atrophy lie either within or very close to the $95 \%$ credible intervals. Our model captures the dynamics of subject 1 similarly well. Only one of the tau data points lies outside the $95 \%$ credible interval, all atrophy data are consistent with the model predictions. When looking at the longterm predictions, the subject exhibits almost linear tau and atrophy dynamics, an observation that is consistent with fairly low transport and growth coefficients inferred for this individual, as discussed in Section 5.2. Our model does a relatively poor job in capturing the dynamics of subjects 2 and 4 . For subject 2, three out of four tau data points and two out of four atrophy data points lie outside the $95 \%$ credible intervals. By design, our model cannot account for non-monotonic increases or decreases in tau data; it always predicts either an overall increasing or an overall decreasing trend for tau. Our model assumptions imply an increase in atrophy whenever the local tau concentration is positive, which results in an overall increase in longitudinal atrophy for subject 2 , even though the observed atrophy does not seem to increase much over time. For subject 4, our model infers an overall declining trend in the global tau concentration from the tau data, again unable to capture the non-monotonically increasing and decreasing data. Consequently, three out of four tau data points lie outside the $95 \%$ credible interval. Even though the observed trend in global atrophy data is overall increasing,
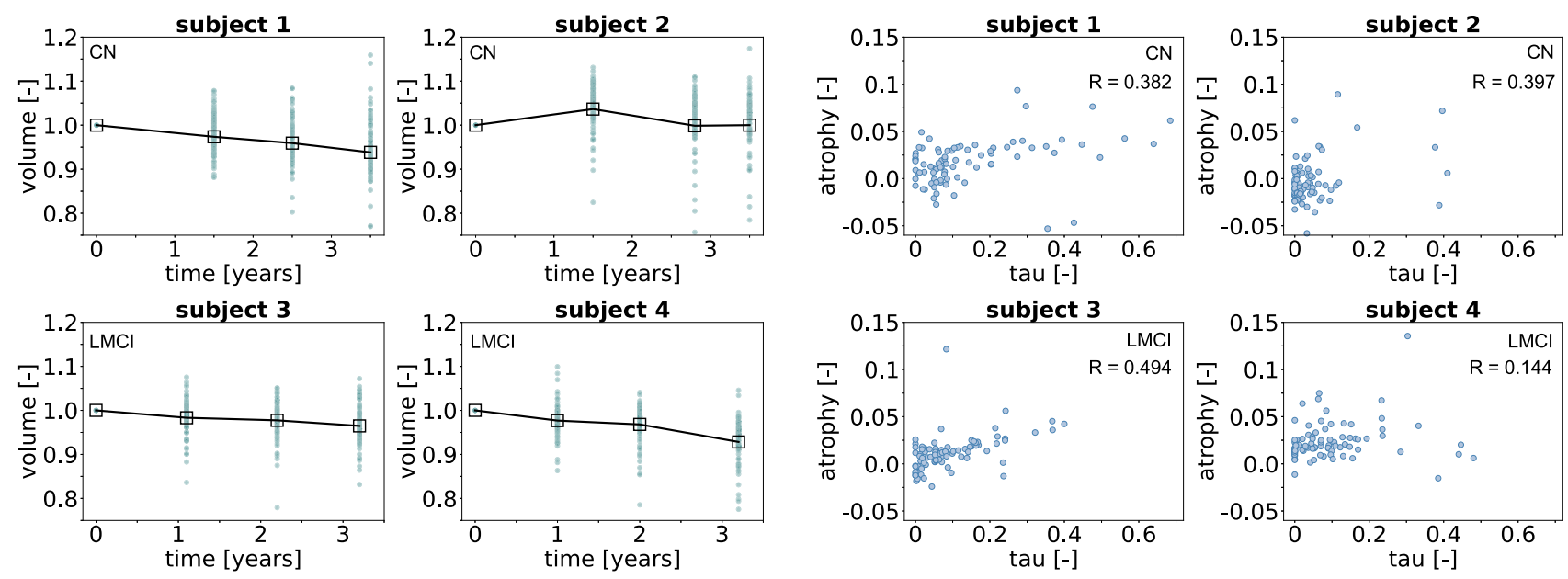

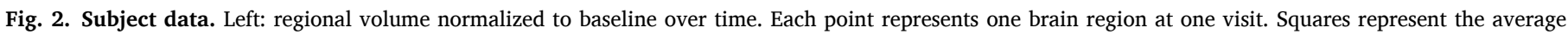

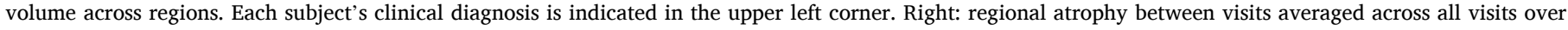
regional tau load averaged across all visits. Each point represents one brain region. R indicates the Pearson correlation coefficient. 


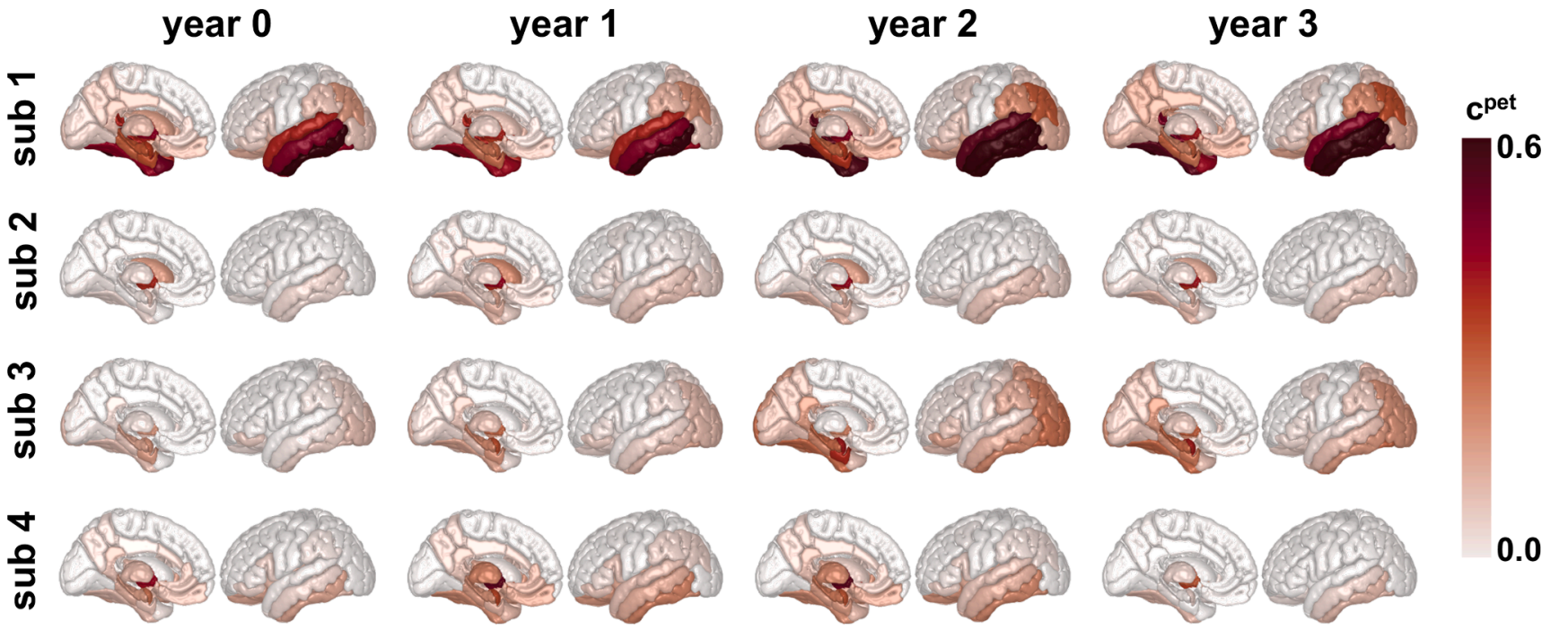

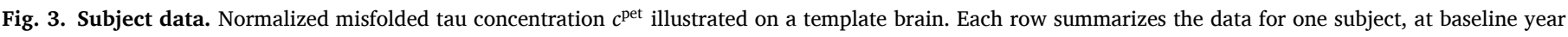
0 and three follow up scans.
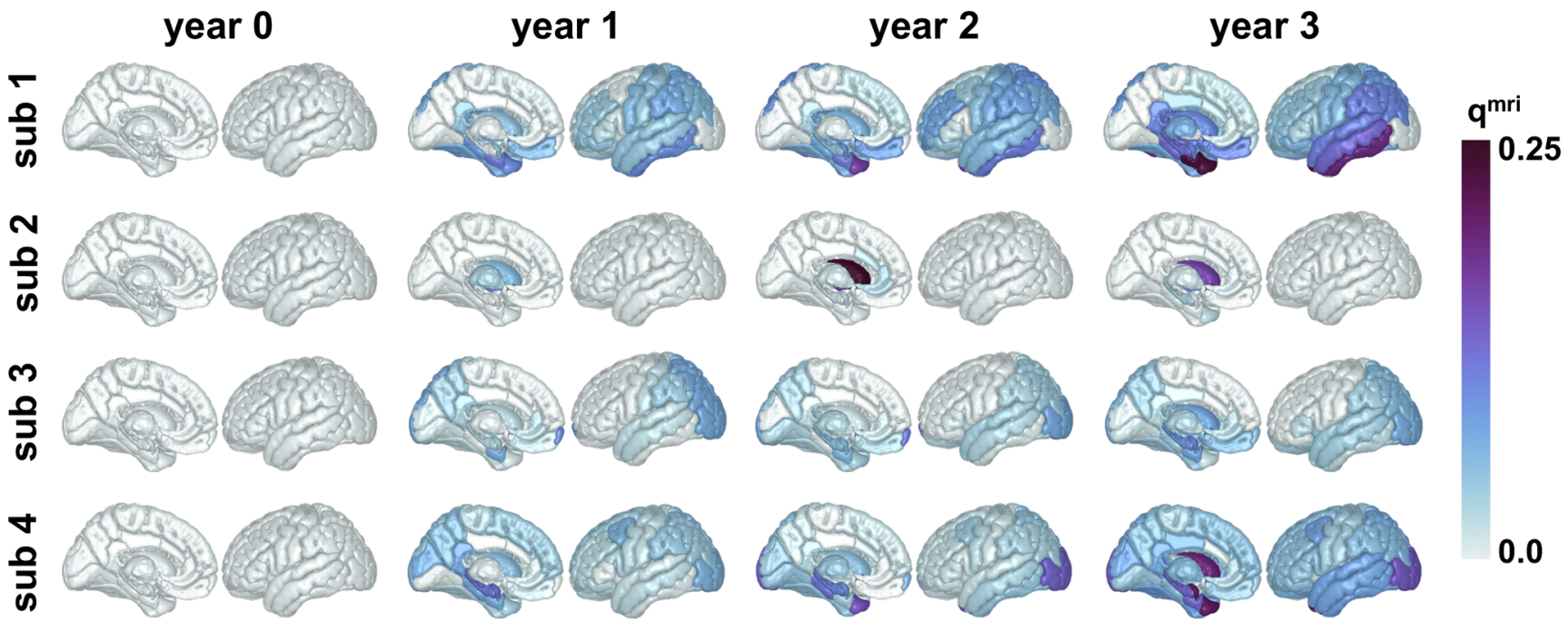

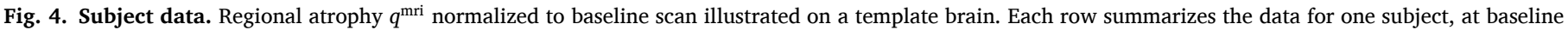
year 0 and three follow up scans.

the inferred negative slope in the tau curve results in a decelerating predicted atrophy rate over time, with whole brain atrophy projected to level out around a value of $25 \%$ after 30 years.

\subsection{Personalized atrophy rates}

We used our posterior predictive simulations to extract predicted time-dependent atrophy rates for the whole brain, the hippocampus, and the entorhinal cortex in each subject. Initial and maximal atrophy rates are presented in Table 3 and may be compared to the observed rates in the literature summarized in Table 1. Fig. 7 shows atrophy rates plotted as a function of time. Subjects 1,2, and 3 exhibit atrophy rates that first increase over time, reach a maximum value, and then decline. We see consistently higher atrophy rates in the hippocampus and the entorhinal cortex than on average across the whole brain, an observation that is in line with the clinical studies summarized in Table 1 . The predicted atrophy rates for subject 1 and subject 2 are in a similar range, while those predicted rates for subject 3 are almost twice as high. Subject 4 exhibits different atrophy dynamics than the rest of the cohort, due to the negative inferred growth coefficient, indicating overall declining tau concentration. The initial atrophy rates inferred for subject 4 are in a similar range as those for subject 1 and subject 2 .

\section{Discussion}

We summarized the characteristics and dynamics of tissue atrophy in Alzheimer's disease and, from the observed trends, proposed a mathematical model that couples misfolded tau propagation and growth with a model of brain atrophy. We performed a Bayesian analysis to personalize our model parameters from longitudinal multi-model imaging data. Posterior predictions allowed us to examine how tau pathology and brain atrophy may develop over three decades in four examined subjects from the Alzheimer's Disease Neuroimaging Initiative. From the posterior predictions, we computed time-dependent annual atrophy rates for each subject and compared these personalized atrophy rates with the values reported in the literature.

The inferred tau-induced atrophy coefficient $G_{c}$, quantifying the effect of local tau on local tissue atrophy, was similar across subjects despite the wide spectrum of observed tau and atrophy dynamics. On average, we found a median tau-induced atrophy coefficient of $G_{c}=$ 

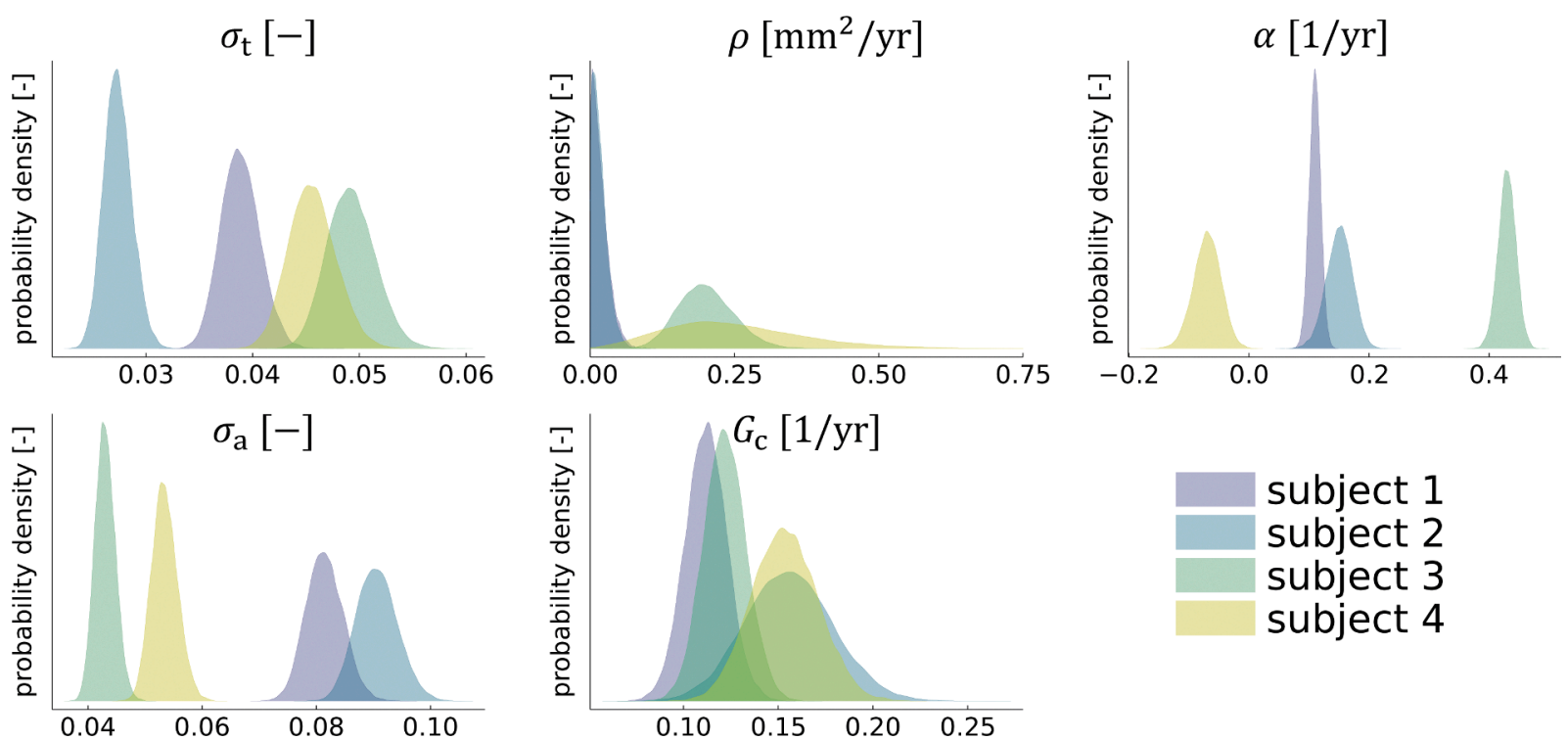

subject 1

subject 2

subject 3

subject 4

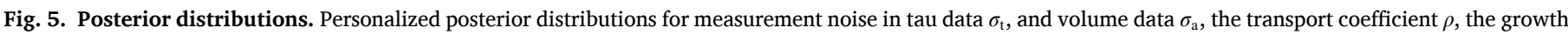
coefficient $\alpha$, and the tau-induced atrophy coefficient $G_{c}$.
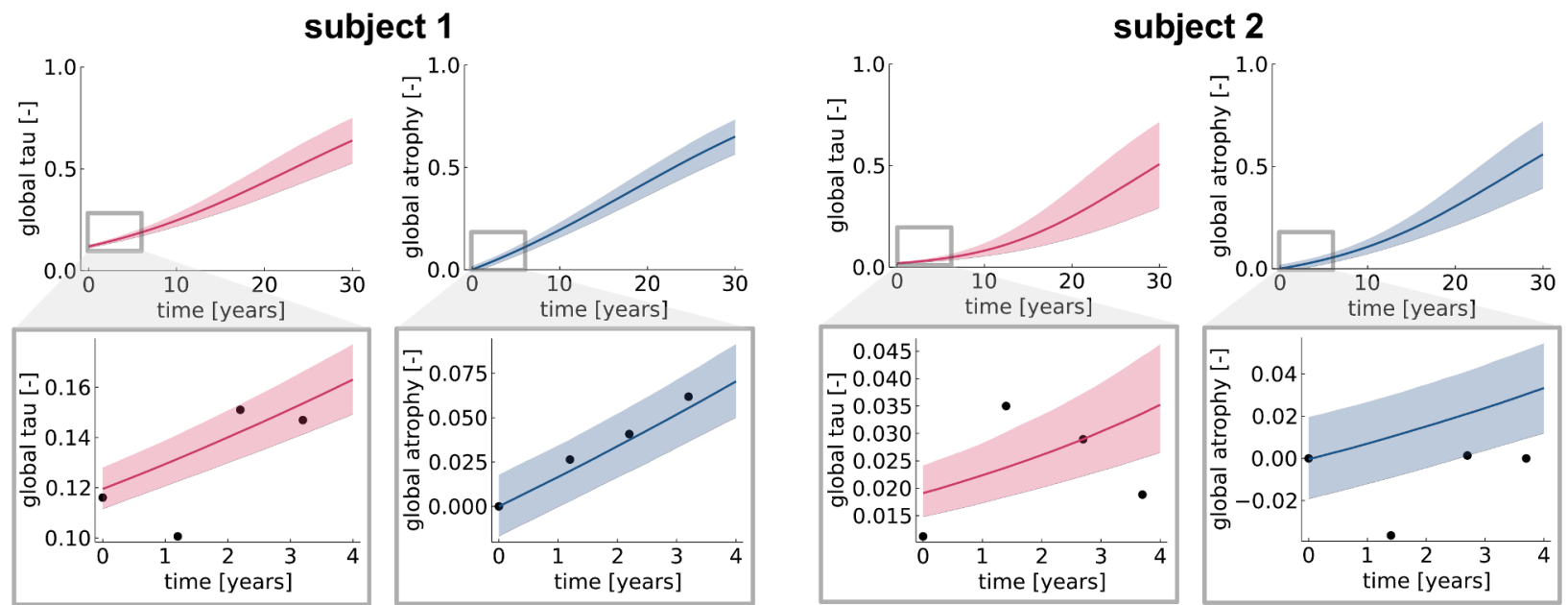

subject 3
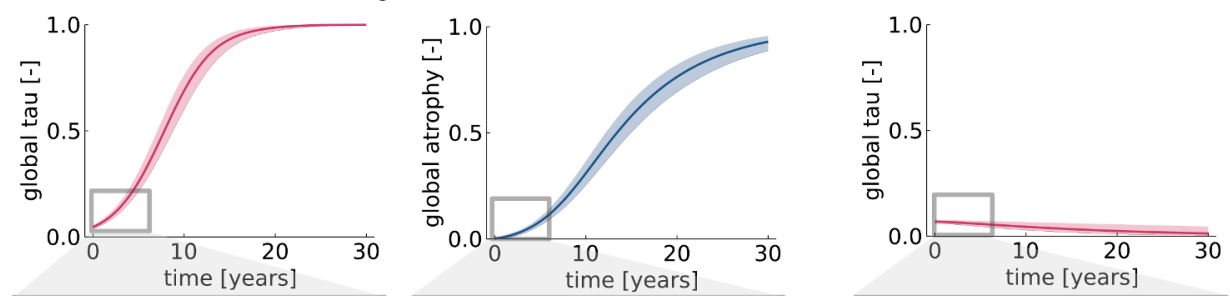

subject 4
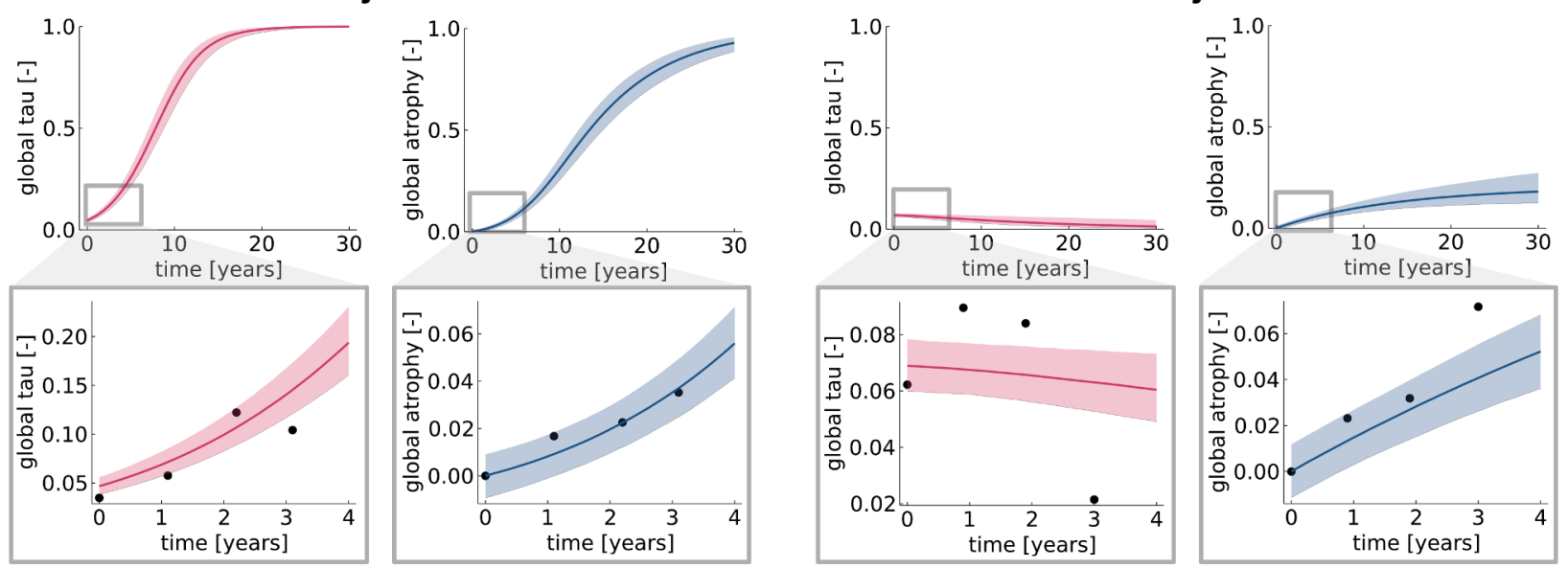

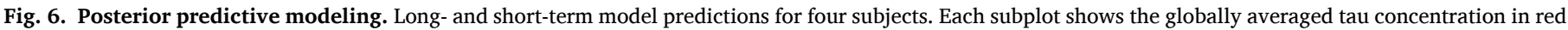

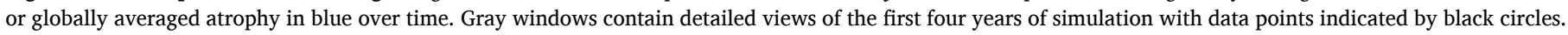

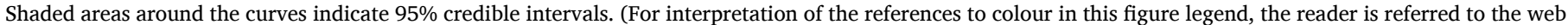
version of this article.) 
Table 3

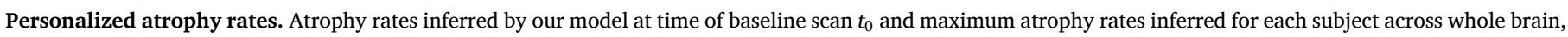
in the hippocampus, and in the entorhinal cortex.

\begin{tabular}{|llllll}
\hline & & \multicolumn{2}{c|}{ predicted atrophy rates: median (95\% CI) in \%/year } \\
& & subject 1 & subject 2 & subject 3 & subject 4 \\
\hline \hline \multirow{2}{*}{ whole brain } & $t_{0}$ & $1.63(1.3-2.0)$ & $0.71(0.5-0.9)$ & $0.68(0.5-0.9)$ & $1.53(1.2-1.9)$ \\
& $\max$ & $2.39(2.0-2.8)$ & $2.61(1.8-3.4)$ & $5.72(5.1-6.3)$ & $1.53(1.2-1.9)$ \\
hippocampus & $t_{0}$ & $2.93(1.2-3.9)$ & $0.39(0.0-1.0)$ & $1.84(1.0-2.8)$ & $3.03(2.0-4.4)$ \\
& $\max$ & $3.62(3.0-4.4)$ & $3.47(1.8-4.6)$ & $5.85(5.2-6.6)$ & $3.03(2.0-4.4)$ \\
entorhinal & $t_{0}$ & $2.53(1.8-3.5)$ & $0.41(0.0-1.0)$ & $2.18(1.3-3.1)$ & $1.26(0.4-2.4)$ \\
cortex & $\max$ & $3.37(2.6-4.1)$ & $2.94(0.7-4.5)$ & $6.4(5.5-7.2)$ & $1.26(0.4-2.4)$ \\
\hline \hline
\end{tabular}
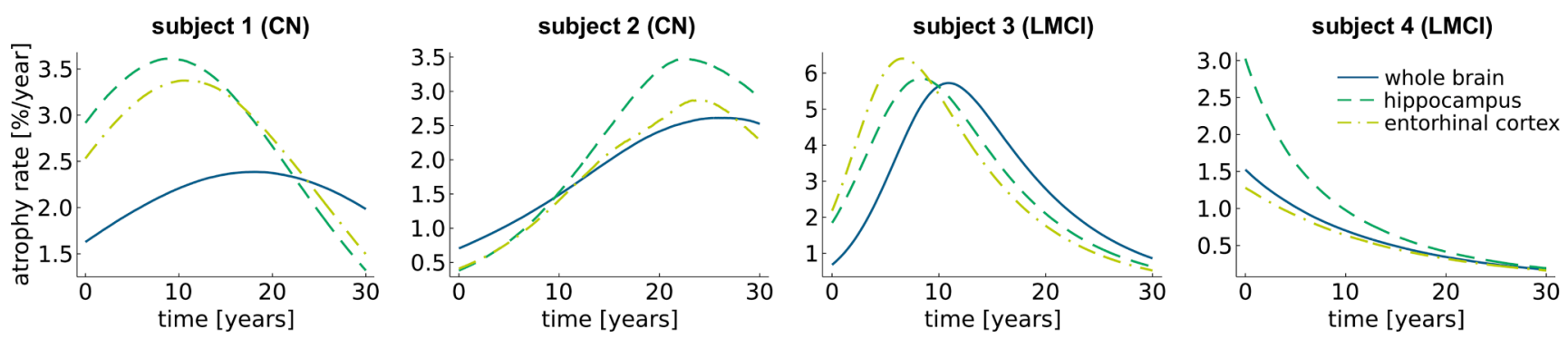

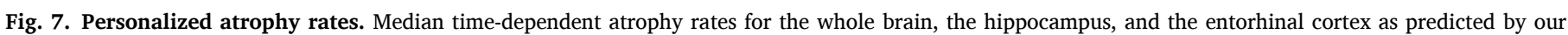
model for each subject.

$0.13 /$ year $(95 \% \mathrm{CI}: 0.097-0.189)$. The consistency in this parameter is a promising sign towards the ability of accurately predicting personalized future atrophy from tau PET images. Developing a predictive model of tau and atrophy dynamics may allow for advances in personalized estimations of disease progression from a set of tau PET images, or estimations of when certain brain functions might be impaired in an individual patient.

The model given by Eqs. 4 and 5 reflects key elements of atrophy dynamics discussed in Section 2, and predicts realistic atrophy rates that are consistent with the values reported in the literature for the whole brain, the hippocampus, and the entorhinal cortex. The whole brain atrophy rates in the literature, as summarized in Table 1 range from $0(0.57) \%$ /year to $0.8(0.5) \%$ /year for control or cognitively normal subjects and from $0.6(0.7) \%$ /year to $2.8(0.5) \%$ /year for subjects with mild cognitive impairment or Alzheimer's disease. Reported hippocampal atrophy rates lie between 0.86 (1.27) \%/year and $3.3(2.4) \%$ /year for cognitively normal subjects and between 2.39 (2.26) \%/year and $5.19(0.75) \%$ year for cognitively impaired patients. For the entorhinal cortex, the reported values range from 0.52 (1.13) \%/year to 5.1 (5.1) \%/year for cognitively normal and from $1.93(1.56) \% /$ year to $8.4(9.2) \% /$ year for cognitively impaired subjects. Apart from subject 4, the plots in Fig. 7 show atrophy rates first increasing, and then decreasing over time, consistent with the observations of early atrophy acceleration and late atrophy deceleration. Additionally, we see consistently higher atrophy rates in the hippocampus and the entorhinal cortex than on average across the whole brain. These two regions are known to be affected by tau and atrophy early in the disease, and their atrophy rates are often used as biomarkers for early diagnosis. Thus, it makes sense that atrophy rates in the hippocampus and entorhinal cortex are higher than average and tend to reach their peak rate earlier than the whole brain on average. For subject 1, the predicted maximum atrophy rates are in good alignment with the rates observed in the literature for patients with Alzheimer's disease or mild cognitive impairment. For a cognitively normal subject, the current diagnosis for subject 1 , the starting atrophy rates are on the higher end compared to the literature, indicating a potential conversion to mild cognitive impairment in the future. This is plausible due to the positive amyloid status associated with subject 1 . Subject 2 exhibits slightly lower beginning atrophy rates, but similar maximum rates as subject 1 , which are also comparable with the literature. For subject 3, our model predicts a steep increase in atrophy rates over the first 10 years after the baseline scan. The predicted maximum whole brain atrophy rate is higher than what is observed on average in the literature, even for Alzheimer's with fast cognitive decline and early onset. The maximum hippocampal and entorhinal atrophy rates are on the high end, but within the range of observed values. Our model predicts declining atrophy rates for subject 4 , starting at about $1.5 \%$ /year for whole brain and entorhinal atrophy rate, and about $3 \%$ year for the hippocampal atrophy rate, and subsequently declining to zero after around 30 years. This trend does not reflect the observations of accelerating atrophy rates described in Section 2, unless this subject has already progressed so far in the disease, that we are only observing the decline in atrophy rates that is described for very late disease stages. The beginning atrophy rates are well in the range of values reported in the literature for whole brain, hippocampus and entorhinal cortex.

This study comes with a number of limitations, the first set of which concern the data used in the study. We constrained our model calibration to a very small, preliminary cohort of four subjects. However, our work demonstrates the feasibility of inferring model parameters from multi-modal data and will be expanded to more subjects to test for the overall statistical significance of our preliminary results. The four examined subjects produced a wide range of tau and atrophy dynamics. Our model does well in explaining the data of subjects 1 and 3 while the results for subjects 2 and 4 suggest that accounting for possible nonmonotony in Eq. 4 may further improve the descriptive capacity of the model. Nevertheless, observing the spectrum of possible dynamics and applicability of the model to even a small preliminary data sample is valuable and highlights the challenges that might be encountered in a larger study. Another limitation is posed by longitudinal data availability. In particular, our data was limited to four time points due to the relative novelty and limited availability of tau PET data in the ADNI database. Specifically, out of the 2492 study participants with imaging data in ADNI, each has on average $4.57 \pm 3.318$ structural MRI scans, but only $0.475 \pm 0.826$ tau PET scans. Only 786 participants have tau 
PET data at all, and out of those each subject has on average $1.506 \pm$ 0.782 longitudinal scans, with a minimum of one scan and maximum of five scans per subject. As tau PET becomes a more established imaging modality across participating study centers, the amount of available longitudinal data will naturally increase, allowing us to improve our model evaluations and assess its predictive capabilities.

The second set of limitations concern simplifications in our model choice. The sparse availability of longitudinal data constrains the number of parameters that that can be reliably inferred while avoiding overfitting. This is an argument for selecting model formulations that balance model expressiveness with data concerns. For instance, the atrophy model presented in Eq. 5 does not account for purely age-related brain atrophy. It is known that age-related atrophy, as discussed in Section 2, and the degradation of white matter integrity [76] both occur in the absence of misfolded tau. White matter degradation is known to alter the fractional anisotropy and mean diffusivity of the axonal bundles. Correlation studies have suggested a link between white matter degradation and atrophy but only a weak relationship to tau [77]. In practice, these observations would suggest coupling the weights of the graph Laplacian, in Eq. 4, to the atrophy variable of Eq. 5. However, this would introduce another set of parameters to the model and strain the available data. Furthermore, this type of coupling has been investigated previously in [78], where it was shown that toxic seeding of tau protein reaches all brain regions before the atrophy variable could have any impact on the graph diffusion. The effect on the disease course was found to be minimal, and arguably negligible, even when the parameters were so extreme as to completely disconnect the network. These results let us to not consider the effect of atrophy on diffusion in our model. However we do note that the effect of atrophy on the graph Laplacian may play a role in more complex models, such as those that take regional clearance into account. Lastly, limited data availability prompted us to exclude any attempt to infer deviations in initial conditions, extracted from the baseline tau PET scan, as a variable in our inference approach. Such an approach would introduce new distributions to be inferred, at every cortical region. The consideration of more complex tau-atrophy models may become more viable as longitudinal data becomes more readily available.

Bayesian methods inherently facilitate working with continuously updated data, a feature that will make it easy to seamlessly update the personalizations with more time points becoming available in the future. In upcoming work, we plan to investigate the identifiability of more complex tau-atrophy models, taking into account the limited amount and inherent noisiness of available imaging data for calibration.

\section{Conclusion}

We presented a high-level overview of what we know, today, about brain atrophy in Alzheimer's disease and demonstrated how we can translate this knowledge into a computational model. Our model captures a coupling between tau pathology and atrophy, and naturally features an early acceleration and late deceleration of atrophy rates. This is made possible through a direct dependence on the local concentration of pathological tau and a saturation term. Personalizing our model with a small data set using a probabilistic approach gives us an initial idea of the ranges of model parameters and associated uncertainties that might occur in real subject data. Strikingly, the magnitude of the tau-induced atrophy coefficient, a direct indicator for the correlation of misfolded tau and tissue atrophy, is fairly consistent across all subjects with a median value of 0.13 year. Using our personalized model, we created long-term predictions for the tau and atrophy dynamics in four subjects and discussed the performance of our model for each individual scenario. We observed that our model generates realistic predictions of atrophy rates that agree well with the rates reported in the literature. Once comprehensively calibrated with a larger set of longitudinal data, our model can be used as a personalized diagnostic and predictive tool to estimate future atrophy from tau PET recordings or serve as a comparative control in treatment studies.

\section{CRediT authorship contribution statement}

Amelie Schäfer: Conceptualization, Methodology, Formal analysis, Writing - original draft. Pavanjit Chaggar: Conceptualization, Methodology, Formal analysis. Travis B. Thompson: Conceptualization, Methodology, Formal analysis, Writing - original draft. Alain Goriely: Conceptualization, Writing - review \& editing. Ellen Kuhl: Conceptualization, Writing - review \& editing.

\section{Declaration of Competing Interest}

The authors declare that they have no known competing financial interests or personal relationships that could have appeared to influence the work reported in this paper.

\section{Acknowledgment}

A. Schäfer was supported by a Brit and Alex d'Arbeloff Stanford Graduate Fellowship to AS. P. Chaggar was supported by funding from the Engineering and Physical Sciences Research Council grant EP/ L016044/1 and Roche. T. Thompson was supported partially through the John Fell Oxford University Press Research Fund grant 000872 (project code BKD00160) to TT, and partially by the Engineering and Physical Sciences Research Council grant EP/R020205/1 to AG. A. Goriely was supported by the Engineering and Physical Sciences Research Council grant EP/R020205/1. E. Kuhl was supported by the National Science Foundation grant CMMI 1727268.

Data collection and sharing for this project was funded by the Alzheimer's Disease Neuroimaging Initiative (ADNI) (National Institutes of Health Grant U01 AG024904) and DOD ADNI (Department of Defense award number W81XWH-12-2-0012). ADNI is funded by the National Institute on Aging, the National Institute of Biomedical Imaging and Bioengineering, and through generous contributions from the following: AbbVie, Alzheimer's Association; Alzheimer's Drug Discovery Foundation; Araclon Biotech; BioClinica, Inc.; Biogen; Bristol-Myers Squibb Company; CereSpir, Inc.; Cogstate; Eisai Inc.; Elan Pharmaceuticals, Inc.; Eli Lilly and Company; EuroImmun; F. Hoffmann-La Roche Ltd and its affiliated company Genentech, Inc.; Fujirebio; GE Healthcare; IXICO Ltd.; Janssen Alzheimer Immunotherapy Research \& Development, LLC.; Johnson \& Johnson Pharmaceutical Research \& Development LLC.; Lumosity; Lundbeck; Merck \& Co., Inc.; Meso Scale Diagnostics, LLC.; NeuroRx Research; Neurotrack Technologies; Novartis Pharmaceuticals Corporation; Pfizer Inc.; Piramal Imaging; Servier; Takeda Pharmaceutical Company; and Transition Therapeutics. The Canadian Institutes of Health Research is providing funds to support ADNI clinical sites in Canada. Private sector contributions are facilitated by the Foundation for the National Institutes of Health (www.fnih.org). The grantee organization is the Northern California Institute for Research and Education, and the study is coordinated by the Alzheimer's Therapeutic Research Institute at the University of Southern California. ADNI data are disseminated by the Laboratory for Neuro Imaging at the University of Southern California.

\section{References}

[1] A. Alzheimer, Uber eine eigenartige erkrankung der hirnrinde, Zentralbl. Nervenh. Psych. 18 (1907) 177-179.

[2] J. Wang, B.J. Gu, C.L. Masters, Y.-J. Wang, A systemic view of alzheimer diseaseinsights from amyloid- $\beta$ metabolism beyond the brain, Nature reviews neurology 13 (10) (2017) 612-623.

[3] S. Bachiller, I. Jiménez-Ferrer, A. Paulus, Y. Yang, M. Swanberg, T. Deierborg, A. Boza-Serrano, Microglia in neurological diseases: a road map to brain-disease dependent-inflammatory response, Front Cell Neurosci 12 (2018) 488.

[4] G.S. Bloom, Amyloid- $\beta$ and tau: the trigger and bullet in alzheimer disease pathogenesis, JAMA Neurol 71 (4) (2014) 505-508. 
[5] J.A. Hardy, G.A. Higgins, Alzheimer'S disease: the amyloid cascade hypothesis, Science 256 (5054) (1992) 184-186.

[6] J. Hardy, D. Allsop, Amyloid deposition as the central event in the aetiology of alzheimer's disease, Trends Pharmacol. Sci. 12 (1991) 383-388.

[7] D.J. Selkoe, J. Hardy, The amyloid hypothesis of alzheimer's disease at 25 years, EMBO Mol Med 8 (6) (2016) 595-608.

[8] A. Bejanin, D.R. Schonhaut, R. La Joie, J.H. Kramer, S.L. Baker, N. Sosa, N. Ayakta, A. Cantwell, M. Janabi, M. Lauriola, et al., Tau pathology and neurodegeneration contribute to cognitive impairment in alzheimer's disease, Brain 140 (12) (2017) 3286-3300.

[9] R. Ossenkoppele, D.R. Schonhaut, M. Schöll, S.N. Lockhart, N. Ayakta, S.L. Baker J.P. O'Neil, M. Janabi, A. Lazaris, A. Cantwell, et al., Tau PET patterns mirror clinical and neuroanatomical variability in alzheimer's disease, Brain 139 (5) (2016) 1551-1567.

[10] T.M. Harrison, R. La Joie, A. Maass, S.L. Baker, K. Swinnerton, L. Fenton, T. J. Mellinger, L. Edwards, J. Pham, B.L. Miller, et al., Longitudinal tau accumulation and atrophy in aging and alzheimer disease, Ann. Neurol. 85 (2) (2019) 229-240.

[11] R. La Joie, A.V. Visani, S.L. Baker, J.A. Brown, V. Bourakova, J. Cha, K. Chaudhary, L. Edwards, L. Iaccarino, M. Janabi, et al., Prospective longitudinal atrophy in alzheimer's disease correlates with the intensity and topography of baseline tauPET, Sci Transl Med 12 (524) (2020).

[12] C. Xia, S.J. Makaretz, C. Caso, S. McGinnis, S.N. Gomperts, J. Sepulcre, T. GomezIsla, B.T. Hyman, A. Schultz, N. Vasdev, et al., Association of in vivo [18f]AV-1451 tau PET imaging results with cortical atrophy and symptoms in typical and atypical alzheimer disease, JAMA Neurol 74 (4) (2017) 427-436.

[13] P.M. Thompson, K.M. Hayashi, G. De Zubicaray, A.L. Janke, S.E. Rose, J. Semple, D. Herman, M.S. Hong, S.S. Dittmer, D.M. Doddrell, et al., Dynamics of gray matter loss in alzheimer's disease, J. Neurosci. 23 (3) (2003) 994-1005.

[14] B.C. Dickerson, A. Bakkour, D.H. Salat, E. Feczko, J. Pacheco, D.N. Greve, F. Grodstein, C.I. Wright, D. Blacker, H.D. Rosas, et al., The cortical signature of alzheimer's disease: regionally specific cortical thinning relates to symptom severity in very mild to mild AD dementia and is detectable in asymptomatic amyloid-positive individuals, Cerebral cortex 19 (3) (2009) 497-510.

[15] G.B. Frisoni, A. Prestia, P.E. Rasser, M. Bonetti, P.M. Thompson, In vivo mapping of incremental cortical atrophy from incipient to overt alzheimer's disease, J. Neurol. 256 (6) (2009) 916-924.

[16] V. Singh, H. Chertkow, J.P. Lerch, A.C. Evans, A.E. Dorr, N.J. Kabani, Spatial patterns of cortical thinning in mild cognitive impairment and alzheimer's disease, Brain 129 (11) (2006) 2885-2893.

[17] J. Vogel, A. Young, N. Oxtoby, O. Hansson, et al., Spread of pathological tau proteins through communicating neurons in human alzheimer's disease, Nat. Med. 11 (2612) (2020).

[18] J. Vogel, Y. Iturria-Medina, O. Hansson, et al., Four distinct trajectories of tau deposition identified in alzheimer's disease, Nat. Commun. 27 (2021) 871-881.

[19] A. Schäfer, E.C. Mormino, E. Kuhl, Network diffusion modeling explains longitudinal tau PET data, Front Neurosci 14 (2020) 1370.

[20] A. Schäfer, M. Peirlinck, K. Linka, E. Kuhl, A.D.N.I. (ADNI, et al., Bayesian physicsbased modeling of tau propagation in alzheimer's disease, Front Physiol (2021) 1081.

[21] J. Weickenmeier, E. Kuhl, A. Goriely, Multiphysics of prionlike diseases: progression and atrophy, Phys. Rev. Lett. 121 (15) (2018) 158101.

[22] A. Schäfer, J. Weickenmeier, E. Kuhl, The interplay of biochemical and biomechanical degeneration in alzheimer's disease, Comput. Methods Appl. Mech. Engrg. 352 (2019) 369-388.

[23] A. Raj, E. LoCastro, M. Weiner, et al., Network diffusion model of progression predicts longitudinal patterns of atrophy and metabolism in alzheimer's disease, Cell Rep 10 (3) (2015) 359-369.

[24] Y. Blinkouskaya, J. Weickenmeier, Brain shape changes associated with cerebral atrophy in healthy aging and alzheimer's disease, Frontiers in Mechanical Engineering (2021) 64.

[25] ADNI, Alzheimer's Disease Neuroimaging Initiative, accessed July 31, 2020 http: //adni.loni.usc.edu.

[26] M. Bobinski, M.J. De Leon, J. Wegiel, S. Desanti, A. Convit, L.A. Saint Louis, H. Rusinek, H.M. Wisniewski, The histological validation of post mortem magnetic resonance imaging-determined hippocampal volume in alzheimer's disease, Neuroscience 95 (3) (1999) 721-725.

[27] K. Im, J.-M. Lee, S.W. Seo, S.H. Kim, S.I. Kim, D.L. Na, Sulcal morphology changes and their relationship with cortical thickness and gyral white matter volume in mild cognitive impairment and alzheimer's disease, Neuroimage 43 (1) (2008) $103-113$.

[28] L. Pini, M. Pievani, M. Bocchetta, D. Altomare, P. Bosco, E. Cavedo, S. Galluzzi, M. Marizzoni, G.B. Frisoni, Brain atrophy in alzheimer's disease and aging, Ageing Res. Rev. 30 (2016) 25-48.

[29] J.M. Burns, J.A. Church, D.K. Johnson, C. Xiong, D. Marcus, A.F. Fotenos, A. Z. Snyder, J.C. Morris, R.L. Buckner, White matter lesions are prevalent but differentially related with cognition in aging and early alzheimer disease, Arch. Neurol. 62 (12) (2005) 1870-1876.

[30] G. Douaud, S. Jbabdi, T.E.J. Behrens, R.A. Menke, A. Gass, A.U. Monsch, A. Rao, B. Whitcher, G. Kindlmann, P.M. Matthews, et al., Dti measures in crossing-fibre areas: increased diffusion anisotropy reveals early white matter alteration in mci and mild alzheimer's disease, Neuroimage 55 (3) (2011) 880-890.

[31] L. O’Dwyer, F. Lamberton, A.L.W. Bokde, M. Ewers, Y.O. Faluyi, C. Tanner, B. Mazoyer, D. O'Neill, M. Bartley, D.R. Collins, et al., Multiple indices of diffusion identifies white matter damage in mild cognitive impairment and alzheimer's disease, PLoS ONE 6 (6) (2011) e21745.
[32] S.M. Nestor, R. Rupsingh, M. Borrie, M. Smith, V. Accomazzi, J.L. Wells, J. Fogarty, R. Bartha, A.D.N. Initiative, Ventricular enlargement as a possible measure of alzheimer's disease progression validated using the alzheimer's disease neuroimaging initiative database, Brain 131 (9) (2008) 2443-2454.

[33] C.R. Jack Jr, F. Barkhof, M.A. Bernstein, M. Cantillon, P.E. Cole, C. DeCarli, B. Dubois, S. Duchesne, N.C. Fox, G.B. Frisoni, et al., Steps to standardization and validation of hippocampal volumetry as a biomarker in clinical trials and diagnostic criterion for alzheimer's disease, Alzheimer's \& Dementia 7 (4) (2011) 474-485.

[34] A.M. Fjell, K.B. Walhovd, C. Fennema-Notestine, L.K. McEvoy, D.J. Hagler, D. Holland, J.B. Brewer, A.M. Dale, One-year brain atrophy evident in healthy aging, J. Neurosci. 29 (48) (2009) 15223-15231.

[35] C. Enzinger, F. Fazekas, P.M. Matthews, S. Ropele, H. Schmidt, S. Smith, R. Schmidt, Risk factors for progression of brain atrophy in aging: six-year followup of normal subjects, Neurology 64 (10) (2005) 1704-1711.

[36] P. Coupé, G. Catheline, E. Lanuza, J.V. Manjón, A.D.N. Initiative, Towards a unified analysis of brain maturation and aging across the entire lifespan: a MRI analysis, Hum Brain Mapp 38 (11) (2017) 5501-5518.

[37] M.R. Sabuncu, R.S. Desikan, J. Sepulcre, B.T.T. Yeo, H. Liu, N.J. Schmansky, M. Reuter, M.W. Weiner, R.L. Buckner, R.A. Sperling, et al., The dynamics of cortical and hippocampal atrophy in alzheimer disease, Arch. Neurol. 68 (8) (2011) 1040-1048.

[38] N.C. Fox, P.A. Freeborough, Brain atrophy progression measured from registered serial MRI: validation and application to alzheimer's disease, J. Magn. Reson. Imaging 7 (6) (1997) 1069-1075.

[39] B.H. Ridha, J. Barnes, J.W. Bartlett, A. Godbolt, T. Pepple, M.N. Rossor, N.C. Fox, Tracking atrophy progression in familial alzheimer's disease: a serial MRI study, The Lancet Neurology 5 (10) (2006) 828-834.

[40] C. Bernard, C. Helmer, B. Dilharreguy, H. Amieva, S. Auriacombe, J.-F. Dartigues, M. Allard, G. Catheline, Time course of brain volume changes in the preclinical phase of alzheimer's disease, Alzheimer's \& Dementia 10 (2) (2014) 143-151.

[41] C.R. Jack, S.D. Weigand, M.M. Shiung, S.A. Przybelski, P.C. O’Brien, J.L. Gunter, D. S. Knopman, B.F. Boeve, G.E. Smith, R.C. Petersen, Atrophy rates accelerate in amnestic mild cognitive impairment, Neurology 70 (19 Part 2) (2008) 1740-1752.

[42] W. Henneman, J.D. Sluimer, J. Barnes, W.M. Van Der Flier, I.C. Sluimer, N.C. Fox, P. Scheltens, H. Vrenken, F. Barkhof, Hippocampal atrophy rates in alzheimer disease: added value over whole brain volume measures, Neurology 72 (11) (2009) 999-1007.

[43] A.B. Zahid, A. Mikheev, N. Srivatsa, J. Babb, U. Samadani, H. Rusinek, Accelerated brain atrophy on serial computed tomography: potential marker of the progression of alzheimer's disease, J Comput Assist Tomogr 40 (5) (2016) 827.

[44] J.D. Sluimer, W.M. van der Flier, G.B. Karas, R. van Schijndel, J. Barnes, R. G. Boyes, K.S. Cover, S.D. Olabarriaga, N.C. Fox, P. Scheltens, et al., Accelerating regional atrophy rates in the progression from normal aging to alzheimer's disease, Eur Radiol 19 (12) (2009) 2826-2833.

[45] C.R. McDonald, L.K. McEvoy, L. Gharapetian, C. Fennema-Notestine, D.J. Hagler, D. Holland, A. Koyama, J.B. Brewer, A.M. Dale, et al., Regional rates of neocortical atrophy from normal aging to early alzheimer disease, Neurology 73 (6) (2009) 457-465.

[46] K.A. Josephs, P.R. Martin, S.D. Weigand, N. Tosakulwong, M. Buciuc, M.E. Murray, L. Petrucelli, M.L. Senjem, A.J. Spychalla, D.S. Knopman, et al., Protein contributions to brain atrophy acceleration in alzheimer's disease and primary agerelated tauopathy, Brain 143 (11) (2020) 3463-3476.

[47] K.K. Leung, J.W. Bartlett, J. Barnes, E.N. Manning, S. Ourselin, N.C. Fox, A.D. N. Initiative, et al., Cerebral atrophy in mild cognitive impairment and alzheimer disease: rates and acceleration, Neurology 80 (7) (2013) 648-654.

[48] J.D. Sluimer, H. Vrenken, M.A. Blankenstein, N.C. Fox, P. Scheltens, F. Barkhof, W. M. Van Der Flier, Whole-brain atrophy rate in alzheimer disease: identifying fast progressors, Neurology 70 (19 Part 2) (2008) 1836-1841.

[49] D. Jacobs, M. Sano, K. Marder, K. Bell, F. Bylsma, G. Lafleche, M. Albert, J. Brandt, Y. Stern, Age at onset of alzheimer's disease: relation to pattern of cognitive dysfunction and rate of decline, Neurology 44 (7) (1994).1215-1215

[50] A.E. Van der Vlies, E. Koedam, Y. Pijnenburg, J. Twisk, P. Scheltens, W.M. Van der Flier, Most rapid cognitive decline in APOE $\varepsilon 4$ negative alzheimer's disease with early onset, Psychol Med 39 (11) (2009) 1907-1911.

[51] D. Chan, J.C. Janssen, J.L. Whitwell, H.C. Watt, R. Jenkins, C. Frost, M.N. Rossor, N.C. Fox, Change in rates of cerebral atrophy over time in early-onset alzheimer's disease: longitudinal MRI study, The Lancet 362 (9390) (2003) 1121-1122.

[52] C.R. Jack, M.M. Shiung, J.L. Gunter, P.C. O'brien, S.D. Weigand, D.S. Knopman, B. F. Boeve, R.J. Ivnik, G.E. Smith, R.H. Cha, et al., Comparison of different MRI brain atrophy rate measures with clinical disease progression in AD, Neurology 62 (4) (2004) 591-600.

[53] J. Barnes, J.W. Bartlett, L.A. van de Pol, C.T. Loy, R.I. Scahill, C. Frost, P. Thompson, N.C. Fox, A meta-analysis of hippocampal atrophy rates in alzheimer's disease, Neurobiol. Aging 30 (11) (2009) 1711-1723.

[54] M.I. Miller, L. Younes, J.T. Ratnanather, T. Brown, H. Trinh, E. Postell, D.S. Lee, M.-C. Wang, S. Mori, R. O'Brien, et al., The diffeomorphometry of temporal lobe structures in preclinical alzheimer's disease, NeuroImage: Clinical 3 (2013) $352-360$.

[55] H. Rusinek, Y. Endo, S. De Santi, D. Frid, W.-H. Tsui, S. Segal, A. Convit, M.J. De Leon, Atrophy rate in medial temporal lobe during progression of alzheimer disease, Neurology 63 (12) (2004) 2354-2359.

[56] J.L. Whitwell, K.A. Josephs, M.E. Murray, K. Kantarci, S.A. Przybelski, S. D. Weigand, P. Vemuri, M.L. Senjem, J.E. Parisi, D.S. Knopman, et al., Mri correlates of neurofibrillary tangle pathology at autopsy: a voxel-based morphometry study, Neurology 71 (10) (2008) 743-749. 
[57] S.R. Das, L. Xie, L.E.M. Wisse, R. Ittyerah, N.J. Tustison, B.C. Dickerson, P. A. Yushkevich, D.A. Wolk, A.D.N. Initiative, et al., Longitudinal and cross-sectiona structural magnetic resonance imaging correlates of AV-1451 uptake, Neurobiol. Aging 66 (2018) 49-58.

[58] T. Timmers, R. Ossenkoppele, E.E. Wolters, S.C.J. Verfaillie, D. Visser, S.S.V. Golla F. Barkhof, P. Scheltens, R. Boellaard, W.M. Van Der Flier, et al., Associations between quantitative [18 f] flortaucipir tau PET and atrophy across the alzheimer's disease spectrum, Alzheimer's research \& therapy 11 (1) (2019) 1-12.

[59] A.N. Kolmogorov, I. Petrovskii, N.S. Piskunov, A study of the equation of diffusion with increase in the quantity of matter, and its application to a biological problem, Byul. Moskovskogo Gos. Univ 1 (6) (1937) 1-25.

[60] J.A. McNab, B.L. Edlow, T. Witzel, S.Y. Huang, H. Bhat, K. Heberlein, T. Feiweier, K. Liu, B. Keil, J. Cohen-Adad, et al., The human connectome project and beyond: initial applications of $300 \mathrm{mt} / \mathrm{m}$ gradients, Neuroimage 80 (2013) 234-245.

[61] T.B. Thompson, P. Chaggar, E. Kuhl, A. Goriely, Protein-protein interactions in neurodegenerative diseases: a conspiracy theory, PLoS Comp. Biol. 16 (10) (2020) e1008267, https://doi.org/10.1371/journal.pcbi.1008267.

[62] C. Kerepesi, B. Szalkai, B. Varga, et al., The braingraph.org database of high resolution structural connectomes and the brain graph tools, Cogn. Neurodyn. 11 (2017) 483-486, https://doi.org/10.1007/s11571-017-9445-1.

[63] The PIT Bioinformatics group, Connectomes: The Braingraph.org public website, 2019, https://braingraph.org/cms/download-pit-group-connectomes/.

[64] P. Putra, P. Chaggar, T. Thompson, A. Goriely, Oxford Mathematical Brain Modelling group: Braid surface Matlab source code and master connectome graphs, 2021, https://github.com/OxMBM/Connectome-Staging.

[65] S.M. Landau, M. Lu, A.D. Joshi, M. Pontecorvo, M.A. Mintun, J.Q. Trojanowski, L. M. Shaw, W.J. Jagust, A.D.N. Initiative, Comparing positron emission tomography imaging and cerebrospinal fluid measurements of $\beta$-amyloid, Ann. Neurol. 74 (6) (2013) 826-836.

[66] S.L. Baker, et al., Reference tissue-based kinetic evaluation of 18F-AV1451 for tau imaging, J. Nucl. Med. 58 (2) (2017) 332-338.

[67] R.S. Desikan, F. Ségonne, B. Fischl, B.T. Quinn, B.C. Dickerson, D. Blacker, R. L. Buckner, A.M. Dale, R.P. Maguire, B.T. Hyman, et al., An automated labeling system for subdividing the human cerebral cortex on MRI scans into gyral based regions of interest, Neuroimage 31 (3) (2006) 968-980.
[68] L. Lemoine, A. Leuzy, K. Chiotis, E. Rodriguez-Vieitez, A. Nordberg, Tau positron emission tomography imaging in tauopathies: the added hurdle of off-target binding, Alzheimer's \& Dementia: Diagnosis, Assessment \& Disease Monitoring 10 (2018) 232-236.

[69] M. Marquié, M.D. Normandin, A.C. Meltzer, M. Siao Tick Chong, N.V. Andrea, A. Antón-Fernández, W.E. Klunk, C.A. Mathis, M.D. Ikonomovic, M. Debnath, et al., Pathological correlations of [F-18]-AV-1451 imaging in non-alzheimer tauopathies, Ann. Neurol. 81 (1) (2017) 117-128.

[70] V.J. Lowe, G. Curran, P. Fang, A.M. Liesinger, K.A. Josephs, J.E. Parisi, K. Kantarci, B.F. Boeve, M.K. Pandey, T. Bruinsma, et al., An autoradiographic evaluation of AV-1451 tau PET in dementia, Acta Neuropathol Commun 4 (1) (2016) 58.

[71] FreeSurfer, FreeSurfer Software Suite, accessed July 31, 2020, http://surfer.nmr. mgh.harvard.edu.

[72] A. Routier, N. Burgos, M. Díaz, M. Bacci, S. Bottani, O. El-Rifai, S. Fontanella, P. Gori, J. Guillon, A. Guyot, et al., Clinica: an open-source software platform for reproducible clinical neuroscience studies, Front Neuroinform 15 (2021).

[73] C. Rackauckas, Q. Nie, Differentialequations.jl-a performant and feature-rich ecosystem for solving differential equations in julia, J Open Res Softw 5 (1) (2017).

[74] H. Ge, K. Xu, Z. Ghahramani, Turing: a language for flexible probabilistic inference. International Conference on Artificial Intelligence and Statistics, AISTATS 2018, 9-11 April 2018, Playa Blanca, Lanzarote, Canary Islands, Spain, 2018, pp. 1682-1690.http://proceedings.mlr.press/v84/ge18b.html

[75] M.D. Homan, A. Gelman, The no-u-turn sampler: adaptively setting path lengths in hamiltonian monte carlo, J. Mach. Learn. Res. 15 (1) (2014) 1593-1623.

[76] D. Madden, I. Bennett, A. Burzynska, G. Potter, N.-K. Chen, A. Song, Diffusion tensor imaging of cerebral white matter integrity in cognitive aging, Biochim. Biophys. Acta Mol. Basis. Dis. 1822 (2012) 386-400.

[77] I. Sintini, C. Schwarz, P. Martin, J. Whitwell, et al., Regional multimodal relationships between tau, hypometabolism, atrophy, and fractional anisotropy in atypical alzheimer's disease, Hum. Brain Mapp. 40 (5) (2019) 1618-1631.

[78] A. Goriely, E. Kuhl, C. Bick, Neuronal oscillations on evolving networks: dynamics, damage, degradation, decline, dementia, and death, Phys. Rev. Lett. 125 (12) (2020) 128102. 